\title{
A CHARACTERIZATION OF THE FIRST HITTING TIME OF DOUBLE INTEGRAL PROCESSES TO CURVED BOUNDARIES
}

\author{
JONATHAN TOUBOUL ${ }^{* * *}$ AND \\ OLIVIER FAUGERAS, ${ }^{* * *}$ Odyssée Laboratory, INRIA/ENS/ENPC
}

\begin{abstract}
The problem of finding the probability distribution of the first hitting time of a double integral process (DIP) such as the integrated Wiener process (IWP) has been an important and difficult endeavor in stochastic calculus. It has applications in many fields of physics (first exit time of a particle in a noisy force field) or in biology and neuroscience (spike time distribution of an integrate-and-fire neuron with exponentially decaying synaptic current). The only results available are an approximation of the stationary mean crossing time and the distribution of the first hitting time of the IWP to a constant boundary. We generalize these results and find an analytical formula for the first hitting time of the IWP to a continuous piecewise-cubic boundary. We use this formula to approximate the law of the first hitting time of a general DIP to a smooth curved boundary, and we provide an estimation of the convergence of this method. The accuracy of the approximation is computed in the general case for the IWP and the effective calculation of the crossing probability can be carried out through a Monte Carlo method.
\end{abstract}

Keywords: First hitting time; first passage time; curved boundary; integrated Wiener process; double integral process; numerical computation

2000 Mathematics Subject Classification: Primary 60G40

Secondary 60G15; 60K40; 65C05

\section{Introduction}

First passage time problems for one-dimensional diffusion processes through a timedependent boundary have received a lot of attention over the last three decades. Unfortunately, the evaluation of the first passage time probability distribution function through a constant or time-dependent boundary is in general an arduous task which has still not received a satisfactory solution. Analytical results are scarce and fragmentary, even if closed-form solutions exist for some very particular cases. Since no analytical method seems to solve the problem, one is led either to the study of the asymptotic behavior of this function and of its moments (see, e.g. [21] and [22]), or to the use of somewhat ad hoc numerical procedures yielding approximate evaluations of the first passage time distributions. Such procedures can be classified as follows: (i) those that are based on probabilistic approaches (see, e.g. [3], [5], [6], [19], [26]), and [27] and (ii) purely numerical methods, such as the widely used Monte Carlo method, which applies without any restriction, but whose results are generally too coarse (for numerical methods, see, e.g. [1], [7], [9], and [13]).

Received 30 August 2007; revision received 24 January 2008.

* Postal address: INRIA, Sophia-Antipolis, 2004 route des Lucioles, BP 93 06902, Sophia-Antipolis Cedex, France.

** Email address: jonathan.touboul@sophia.inria.fr

*** Email address: olivier.faugeras@sophia.inria.fr 
In two and higher dimensions, the problem is even more complex and results can hardly be found. For the simplest double integral process (DIP), the integrated Wiener process (IWP) defined in (5), below, McKean [18], Goldman [10], and Lachal [14], [15], [16] found the probability distribution of the first hitting time to a constant boundary using stochastic calculus methods. Lefebvre [17] used the Kolmogorov (Fokker-Planck) equation to find in some special cases closed-form solutions. Generalizations of these formulae to other boundaries and other kinds of processes are simply not available. In the present paper we propose a closed-form solution for the first hitting time of the IWP to a piecewise-cubic function, and apply this formula to find an approximation of the first hitting time of a DIP to any smooth curved boundary. We also provide an estimation of the rate of convergence of this approximation.

In Section 2 we introduce a motivation of this study, define the DIP, and prove the main properties which will be useful for us in the rest of the paper. In Section 3 we study the first hitting times of the IWP and provide a closed-form formula for the first hitting time of this process to a piecewise-cubic function. In Section 4 we introduce the approximation method of the first hitting time of the IWP to any smooth curved boundary, and find the rate of convergence of this method. Finally, in Section 5 we provide an approximation formula for the first hitting time of a general DIP to a curved boundary. In Section 6 we briefly describe a numerical Monte Carlo algorithm which can be used to compute the probability repartition function efficiently.

\section{The DIP}

In this section we introduce the DIP and prove some useful properties. But before the mathematical study of the problem, we motivate this theoretical work by a specific problem arising in neuroscience: the distribution of the spike times for an integrate-and-fire neuron with exponentially decaying synaptic currents.

\subsection{Motivation}

The definition of the DIP and the study of its first hitting times to curved boundaries has been motivated by numerous physical and biological problems. For instance, a problem arising in neuroscience is to characterize the probability distribution of the spike (action potentials) times in the presence of synaptic noise (see [8] for an introduction of the neuronal modelization of spiking neurons and [30] for a review of the problem of spike time distribution).

A classical neuron model is the leaky integrate-and-fire model, where the membrane potential $V(t)$ of a neural cell integrates external inputs and the noise at the synapses, and emits a spike when the membrane potential reaches a deterministic threshold function $\theta(t)$ (which is constant in general). Hence, in this model the membrane potential is the solution of the following equation:

$$
\tau_{\mathrm{m}} \mathrm{d} V(t)=\left(-\left(V(t)-V_{\text {rest }}\right)+I_{\mathrm{e}}(t)\right) \mathrm{d} t+\mathrm{d} I_{\mathrm{S}}(t) .
$$

In this equation $\tau_{\mathrm{m}}$ is the characteristic time of integration of the membrane potential, $V_{\text {rest }}$ is the rest potential of the neuron, $I_{\mathrm{e}}$ represents deterministic external inputs, and $I_{\mathrm{S}}$ represents the noisy synaptic inputs (see, e.g. [8] and [30]). The simplest model of synaptic noise is a standard Brownian motion, if we neglect the integration time of the synapse. Nevertheless, real post-synaptic currents have a very short rise time and a larger decay time.

If we take into account the decay time of the synapse $\tau_{\mathrm{s}}$ then the synaptic current is the solution of the following stochastic differential equation:

$$
\tau_{\mathrm{S}} \mathrm{d} I_{\mathrm{S}}(t)=-I_{\mathrm{S}}(t) \mathrm{d} t+\sigma \mathrm{d} W_{t},
$$




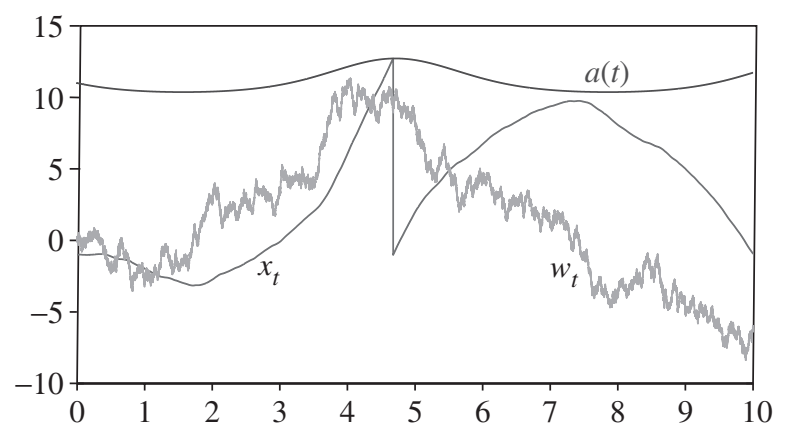

Figure 1: A sample path of the process $U_{t}=\left(X_{t}, W_{t}\right)$, where $X$ is a standard IWP and $W$ is a standard Brownian motion, and a boundary curve $a(t)$. The IWP $X_{t}$ is reset to 0 when it crosses the boundary.

where $\sigma>0$ is the noise standard deviation. We can integrate this system of stochastic differential equations as follows. The equation governing the membrane potential yields

$V(t)=V_{\text {rest }}\left(1-\exp \left(-\frac{t}{\tau_{\mathrm{m}}}\right)\right)+\frac{1}{\tau_{\mathrm{m}}} \int_{0}^{t} \exp \left(\frac{s-t}{\tau_{\mathrm{m}}}\right) I_{\mathrm{e}}(s) \mathrm{d} s+\frac{1}{\tau_{\mathrm{m}}} \int_{0}^{t} \exp \left(\frac{s-t}{\tau_{\mathrm{m}}}\right) I_{\mathrm{s}}(s) \mathrm{d} s$,

and the synaptic current equation can be integrated as

$$
I_{\mathrm{S}}(t)=I_{\mathrm{S}}(0) \exp \left(-\frac{t}{\tau_{\mathrm{S}}}\right)+\frac{\sigma}{\tau_{\mathrm{S}}} \int_{0}^{t} \exp \left(\frac{s-t}{\tau_{\mathrm{S}}}\right) \mathrm{d} W_{s}
$$

where $I_{\mathrm{S}}(0)$ is a given random variable. We define $1 / \alpha=1 / \tau_{\mathrm{m}}-1 / \tau_{\mathrm{s}}$. Replacing $I_{\mathrm{S}}(t)$ in the first equation by its value in the second equation we obtain

$$
\begin{aligned}
V(t)= & V_{\text {rest }}\left(1-\exp \left(-\frac{t}{\tau_{\mathrm{m}}}\right)\right)+\frac{1}{\tau_{\mathrm{m}}} \int_{0}^{t} \exp \left(\frac{s-t}{\tau_{\mathrm{m}}}\right) I_{\mathrm{e}}(s) \mathrm{d} s \\
& +\frac{I_{\mathrm{s}}(0)}{1-\tau_{\mathrm{m}} / \tau_{\mathrm{s}}}\left(\exp \left(-\frac{t}{\tau_{\mathrm{s}}}\right)-\exp \left(-\frac{t}{\tau_{\mathrm{m}}}\right)\right) \\
& +\frac{\sigma}{\tau_{\mathrm{m}} \tau_{\mathrm{s}}} \exp \left(-\frac{t}{\tau_{\mathrm{m}}}\right) \int_{0}^{t} \mathrm{e}^{s / \alpha}\left(\int_{0}^{s} \exp \left(\frac{s^{\prime}}{\tau_{\mathrm{s}}}\right) \mathrm{d} W_{s^{\prime}}\right) \mathrm{d} s
\end{aligned}
$$

The time of the spike emission is the first hitting time of $V(t)$ to the threshold $\theta(t)$; hence, it is the first hitting time of the stochastic process (which is a particular case of what we will call in the sequel the double integral process (DIP))

$$
X_{t}=\int_{0}^{t} \mathrm{e}^{s / \alpha}\left(\int_{0}^{s} \exp \left(\frac{s^{\prime}}{\tau_{\mathrm{s}}}\right) \mathrm{d} W_{s^{\prime}}\right) \mathrm{d} s
$$

to the deterministic curved boundary

$$
\begin{gathered}
a(t)=\theta(t)-\left(V_{\text {rest }}\left(1-\exp \left(-\frac{t}{\tau_{\mathrm{m}}}\right)\right)+\frac{1}{\tau_{\mathrm{m}}} \int_{0}^{t} \exp \left(\frac{s-t}{\tau_{\mathrm{m}}}\right) I_{\mathrm{e}}(s) \mathrm{d} s\right. \\
\left.+\frac{I_{\mathrm{s}}(0)}{1-\tau_{\mathrm{m}} / \tau_{\mathrm{s}}}\left(\exp \left(-\frac{t}{\tau_{\mathrm{s}}}\right)-\exp \left(-\frac{t}{\tau_{\mathrm{m}}}\right)\right)\right)
\end{gathered}
$$

(see Figure 1). 


\subsection{Definition and main properties of DIPs}

In this subsection we define a class of stochastic processes including process (1), and prove some useful properties of these processes.

Definition 1. (DIP.) Let $f \in \mathbb{L}^{2}(\mathbb{R})$ and $g \in \mathbb{L}^{1}(\mathbb{R})$. Let $M_{t}$ be the martingale defined by $M_{t}:=\int_{0}^{t} f(s) \mathrm{d} W_{s}$. The DIP associated to the functions $f$ and $g$ is defined, for all $t$, by

$$
X_{t}=\int_{0}^{t} g(s) M_{s} \mathrm{~d} s=\int_{0}^{t} g(s)\left(\int_{0}^{s} f(u) \mathrm{d} W_{u}\right) \mathrm{d} s .
$$

Proposition 1. The two-dimensional process $\left(X_{t}, M_{t}\right)$ is a Gaussian Markov process.

Proof. First of all, note that if $\mathcal{F}_{t}^{X}$ and $\mathcal{F}_{t}{ }^{M}$ define the canonical filtration associated to the processes $X$ and $M$, respectively, then it is clear that, for all $t \geq 0, \mathcal{F}_{t}^{X} \subset \mathcal{F}_{t}^{M}$. Hence, the filtration associated to the pair $\left(X_{t}, M_{t}\right)_{t \geq 0}$ is simply $\left(\mathcal{F}_{t}^{M}\right)_{t \geq 0}$, which we denote by $\left(\mathcal{F}_{t}\right)_{t \geq 0}$ in the sequel.

It is also clear that $M$ is a martingale, and satisfies the Markov property. Let $s \leq t$. We have

$$
\begin{gathered}
X_{t}=\int_{0}^{t} g(u) M_{u} \mathrm{~d} u=\int_{0}^{s} g(u) M_{u} \mathrm{~d} u+\int_{s}^{t} g(u) M_{u} \mathrm{~d} u, \\
X_{t}=X_{s}+\int_{s}^{t} g(u)\left(M_{u}-M_{s}\right) \mathrm{d} u+M_{s} \int_{s}^{t} g(u) \mathrm{d} u .
\end{gathered}
$$

Conditionally to $M_{s}$, the process $\int_{s}^{t} g(u)\left(M_{u}-M_{s}\right) \mathrm{d} u$ is independent of $\mathcal{F}_{s}^{M}$, so the law of $X_{t}$, knowing $\left(X_{s}, M_{s}\right)$, is independent of the $\sigma$-algebra $\left(\mathcal{F}_{t}\right)$, and so is $M$, so eventually the pair $(X, M)$ is Markov.

The pair is clearly a Gaussian process since its two components are. Indeed, $M$ is Gaussian because of the properties of the stochastic integral seen as the limit of the Riemann sums of Brownian increments, and $X$, as the limit of Riemann sums of the Gaussian process $M$ weighted by $g$, is also Gaussian.

Remark 1. In the proof of Proposition 1 we also proved that, conditionally to $M_{s}$, the increments $\left(X_{t}-X_{s}, M_{t}-M_{s}\right)$ are independent of the $\sigma$-field $\mathcal{F}_{s}$.

Proposition 2. For each value of $t \geq 0$, the random variable $Y_{t}:=\left(X_{t}, M_{t}\right)$ is a twodimensional Gaussian variable of parameters

$$
\begin{gathered}
\mathrm{E}\left[Y_{t}\right]=(0,0), \\
\mathrm{E}\left[Y_{t}^{\top} Y_{t}\right]=\left(\begin{array}{cc}
\rho_{X}(0, t) & C_{(X, M)}(0, t) \\
C_{(X, M)}(0, t) & \rho_{M}(0, t)
\end{array}\right),
\end{gathered}
$$

where the functions $\rho_{X}(s, t), C_{(X, M)}(s, t)$, and $\rho_{M}(s, t)$ are defined by

$$
\begin{gathered}
\rho_{M}(s, t)=\int_{s}^{t} f(u)^{2} \mathrm{~d} u, \\
\rho_{X}(s, t)=2 \int_{s}^{t} g(u)\left(\int_{s}^{u} g(v) \rho_{M}(s, v) \mathrm{d} v\right) \mathrm{d} u, \\
C_{(X, M)}(s, t)=\int_{s}^{t} g(u) \rho_{M}(s, u) \mathrm{d} u .
\end{gathered}
$$


The transition measure of the Markov process $\left(Y_{t}\right)_{t}$ has a Gaussian density with respect to Lebesgue's measure:

$$
\mathcal{N}\left(\left(\begin{array}{c}
x_{s}+m_{s} \int_{s}^{t} g(u) \mathrm{d} u \\
m_{s}
\end{array}\right), \tilde{C}(s, t)\right),
$$

where the correlation matrix $\tilde{C}(s, t)$ reads

$$
\tilde{C}(s, t)=\left(\begin{array}{cc}
\rho_{X}(s, t) & C_{(X, M)}(s, t) \\
C_{(X, M)}(s, t) & \rho_{M}(s, t) \mathrm{d} u
\end{array}\right) .
$$

Proof. The calculations are essentially straightforward. To compute the transition density function, we use (3) and write

$$
\left(\begin{array}{l}
X_{t} \\
M_{t}
\end{array}\right)=\left(\begin{array}{c}
X_{s}+M_{s} \int_{s}^{t} g(u) \mathrm{d} u \\
M_{s}
\end{array}\right)+\left(\begin{array}{c}
\int_{s}^{t} g(u)\left(M_{u}-M_{s}\right) \mathrm{d} u \\
M_{t}-M_{s}
\end{array}\right) .
$$

The first term in the sum on the right-hand side of (4) is $\mathcal{F}_{s}$ measurable. Given $X_{s}=x_{s}$ and $M_{s}=m_{s}$, it is equal to

$$
\left(\begin{array}{c}
x_{s}+m_{s} \int_{s}^{t} g(u) \mathrm{d} u \\
m_{s}
\end{array}\right) \text {. }
$$

The second term is independent of $\mathscr{F}_{s}$ and is Gaussian.

Eventually, the process $Y_{t}$, knowing $Y_{s}=\left(x_{s}, m_{s}\right)$, has the same law as the Gaussian process:

$$
\mathcal{N}\left(\left(\begin{array}{c}
x_{s}+m_{s} \int_{s}^{t} g(u) \mathrm{d} u \\
m_{s}
\end{array}\right), \tilde{C}(s, t)\right) .
$$

Definition 2. (IWP.) The IWP is a special case of the DIP where the functions $f$ and $g$ are identically equal to 1 :

$$
X_{t}=\int_{0}^{t} W_{s} \mathrm{~d} s, \quad M_{s}=W_{s} .
$$

From Proposition 2 we know that its transition measure reads

$$
\begin{aligned}
\mathrm{P}\left[X_{t+s}\right. & \left.\in \mathrm{d} u, W_{t+s} \in \mathrm{d} v \mid X_{s}=x, W_{s}=y\right] \\
& :=p_{t}(u, v ; x, y) \mathrm{d} u \mathrm{~d} v \\
& =\frac{\sqrt{3}}{\pi t^{2}} \exp \left(-\frac{6}{t^{3}}(u-x-t y)^{2}+\frac{6}{t^{2}}(u-x-t y)(v-y)-\frac{2}{t}(v-y)^{2}\right) \mathrm{d} u \mathrm{~d} v .
\end{aligned}
$$

Lemma 1. Let $\left(X_{t}\right)_{t \geq 0}$ be a DIP defined by (2). Assume that $f(s) \neq 0$ for all $s \geq 0$. The study of the hitting times of the DIP $X$ is equivalent to the study of the simpler process

$$
\tilde{X}_{t}=\int_{0}^{t} \tilde{g}(s) W_{s} \mathrm{~d} s
$$

where $\tilde{g}$ is defined in the proof.

Proof. Let $\left(M_{t}\right)_{t}$ be the martingale defined by

$$
M_{t}=\int_{0}^{t} f(s) \mathrm{d} W_{s} .
$$


The Dubins-Schwarz theorem ensures us (even though $\langle M\rangle_{\infty} \neq \infty$, because of our hypothesis on $f$ (see [12])) that there exists a Brownian motion $\left(W_{t}\right)_{t}$ such that, almost surely,

$$
M_{t}=W_{\langle M\rangle_{t}},
$$

where $\langle Z\rangle_{t}$ denotes the quadratic variation of the local martingale $Z$.

We note that

$$
\Phi(t)=\langle M\rangle_{t}=\int_{0}^{t} f^{2}(s) \mathrm{d} s .
$$

The function $\Phi$ is continuous and since we assumed that $f(s) \neq 0$ for all $s \geq 0$, strictly increasing, so it is a bijection. Its derivative $\Phi^{\prime}(t)$ exists and is nonzero for all $t \geq 0$. We use the change of variable $u=\Phi(s)$. We have

$$
\begin{aligned}
X_{t} & =\int_{0}^{t} g(s) M_{s} \mathrm{~d} s \\
& \stackrel{\mathcal{L}}{=} \int_{0}^{t} g(s) W_{\Phi(s)} \mathrm{d} s \\
& =\int_{0}^{\Phi^{-1}(t)} \frac{g\left(\Phi^{-1}(u)\right)}{\Phi^{\prime}\left(\Phi^{-1}(u)\right)} W_{u} \mathrm{~d} u,
\end{aligned}
$$

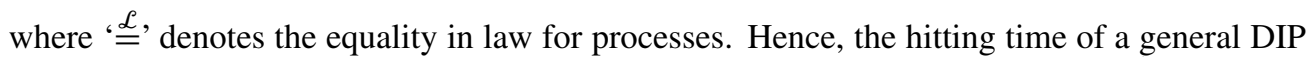
can be deduced from the hitting time of the process $\tilde{X}_{t}=X_{\Phi(t)}$, which is of type $\int_{0}^{t} \tilde{g}(s) W_{s} \mathrm{~d} s$, where $\tilde{g}(t)=g\left(\Phi^{-1}(t)\right) / \Phi^{\prime}\left(\Phi^{-1}(t)\right)$.

\section{First hitting time of the IWP}

We consider the special case $\left(W_{t}\right)_{t \geq 0}$, a standard Brownian motion. We are interested in the first hitting time to a curved boundary $a(t)$ of the stochastic process

$$
X_{t}=\int_{0}^{t} W_{s} \mathrm{~d} s .
$$

This problem has been widely studied and has received no satisfactory solution so far. One of the main difficulties comes from the fact that the process is non Markov, so we have to refer to the underlying Wiener process. Classical approaches based on Volterra equations or Durbin's method work for the Brownian motion, but fail in providing a solution to this problem (see, e.g. [30] for a review). To achieve the program of characterizing those hitting times, we first recall existing results on the first hitting times to constant boundaries, and generalize them to cubic and piecewise-cubic boundaries, to end with the approximation formula for general boundaries.

\subsection{First hitting time to a constant boundary}

Lachal [14] studied this problem in the case where the boundary is a constant. More precisely, in this subsection we study the process $U_{t}=\left(X_{t}+x+t y, W_{t}+y\right)$, where $X_{t}$ is the standard IWP. We denote by

$$
\tau_{a}=\inf \left\{t>0 ; X_{t}+x+t y=a\right\}
$$

the first passage time at $a$ of the first component of the bidimensional Markov process $U_{t}$. The work of Lachal [14] followed the work of McKean [18], where the joint law of the process 
$\left(\tau_{a}, W_{\tau_{a}}\right)$ was computed for the case in which $x=a$. The result was

$$
\begin{aligned}
\mathrm{P}\left[\tau_{a}\right. & \left.\in \mathrm{d} t ;\left|W_{\tau_{a}}\right| \in \mathrm{d} z \mid U_{0}=(a, y)\right] \\
& :=\mathrm{P}_{(a, y)}\left[\tau_{a} \in \mathrm{d} t ;\left|W_{\tau_{a}}\right| \in \mathrm{d} z\right] \\
& =\frac{3 z}{\pi \sqrt{2} t^{2}} \mathrm{e}^{-2 / t}\left(y^{2}-|y| z+z^{2}\right)\left(\int_{0}^{4|y| z / t} \mathrm{e}^{-3 \theta / 2} \frac{\mathrm{d} \theta}{\sqrt{\pi \theta}}\right) \mathbf{1}_{[0,+\infty)}(z) \mathrm{d} z \mathrm{~d} t .
\end{aligned}
$$

We denote this density by $m^{a}(t, y, z)$.

Later, Goldman [10] computed the distribution of the random variable $\tau_{a}$ in the case where $x<a$ and $y \leq 0$, and obtained the formula

$$
\begin{aligned}
\mathrm{P}\left[\tau_{a} \in \mathrm{d} t\right. & \left.\mid U_{0}=(x, y)\right] \\
=\mathrm{d} t & \left(\sqrt{\frac{3}{8 \pi t^{3}}\left(\frac{3(a-x)}{t}-y\right) \exp \left(-\frac{3(a-x-t y)^{2}}{2 t^{3}}\right)}\right. \\
& \left.\quad+\int_{0}^{+\infty} z \mathrm{~d} z \int_{0}^{t} \int_{0}^{\infty} \mathrm{P}\left[\tau_{0} \in \mathrm{d} s ;\left|W_{\tau_{0}}\right| \in \mathrm{d} \mu \mid U_{0}=(0, z)\right] q_{t-s}(x, y ; a, z)\right),
\end{aligned}
$$

where $q_{t}(x, y ; u, v)=p_{t}(x, y ; u, v)-p_{t}(x, y ; u,-v)$.

Lastly, Lachal [14] extended all these results and gave the joint distribution of the pair $\left(\tau_{a}, W_{\tau_{a}}\right)$ in all cases. The quite complex formula reads

$$
\begin{aligned}
\mathrm{P}_{(x, y)} & {\left[\tau_{a} \in \mathrm{d} t ; W_{\tau_{a}} \in \mathrm{d} z\right] } \\
& =|z|\left(p_{t}(x, y ; a, z)-\int_{0}^{t} \int_{0}^{+\infty} m^{0}(s,-|z|, \mu) p_{t-s}(x, y ; a,-\varepsilon \mu) \mathrm{d} \mu \mathrm{d} s\right) \mathbf{1}_{A}(z) \mathrm{d} z \mathrm{~d} t
\end{aligned}
$$

where $A=[0, \infty)$ if $x<a, A=(-\infty, 0]$ if $x>a, \varepsilon=\operatorname{sgn}(a-x)$, and $m^{0}(s,-|z|, \mu)$ is given by McKean's formula, (7). We denote this density by $l_{x, y}^{a}(t, z)$.

\subsection{First hitting time to a cubic boundary}

The problem we address now is the question of finding similar formulae for more general boundaries. For the Brownian motion itself, few results are available. A formula has been found for a linear boundary using Girsanov's theorem and for a quadratic boundary using the Laplace transform characterization (see [11]). Lastly, the method of images has been shown to provide closed-form results in very particular cases (see [24] for a review). The difficulty of finding closed-form characterizations of the first hitting time of the Brownian motion motivated people to look for approximations. Monte Carlo simulation is often used. Even if it can be used with no restriction, it is often considered too coarse and computationally inefficient. Furthermore, it is purely numerical and global, and does not provide any analytical information on the hitting time. For these reasons, other semi-analytical methods of approximation have been developed to provide analytical approximations [4], [5], [6], [25], [31], sometimes together with error estimations [2], [23].

The problem is even more complex for the first hitting time of the IWP.

In this subsection we apply Girsanov's theorem to transform the problem of finding a closedform expression of the first hitting time of the IWP to a cubic function to the problem discussed in the previous subsection that has been solved by McKean [18], Goldman [10], and Lachal [14]. More precisely, we prove that, under a certain probability, the process $W_{t}+(\beta / 2) t^{2}+\alpha t+x$ for 
any $\alpha, \beta \in \mathbb{R}$ is a Wiener process. Under this probability, the process $X_{t}+(\beta / 6) t^{3}+(\alpha / 2) t^{2}+$ $t x+y$ has the law of an IWP. Hence, the knowledge of the probability density function (PDF) of the first hitting time of the IWP to a constant will give us, using Girsanov's theorem, the PDF of the hitting time of the IWP to the cubic

$$
t \mapsto \frac{\beta}{6} t^{3}+\frac{\alpha}{2} t^{2}+b t+a .
$$

For the sake of generality, we compute the new probability starting at a general time $s$ at the point $(x, y)$. In the following the index $\{s,(x, y)\}$ denotes the conditioning on the event $\left(X_{s}=x, W_{s}=y\right)$.

Theorem 1. The process $W_{t}+\alpha(t-s)+(\beta / 2)(t-s)^{2}+y, 0 \leq s \leq t$, is a Wiener process starting at $y$ at time $s$, under the probability

$$
\left.\mathrm{P}_{s,(x, y)}^{\alpha, \beta}\right|_{\mathcal{F}_{s, t}}=\left.D_{s,(x, y)}^{\alpha, \beta}(t) \mathrm{P}_{s,(x, y)}\right|_{\mathcal{F}_{s, t}},
$$

where

$$
\begin{aligned}
D_{s,(x, y)}^{\alpha, \beta}(t)=\exp ( & -\frac{1}{6} \beta^{2}\left(t^{3}-s^{3}\right)-\frac{1}{2} \alpha \beta\left(t^{2}-s^{2}\right)-\frac{1}{2} \alpha^{2}(t-s) \\
& \left.-(\alpha+t \beta) W_{t}+(\alpha+s \beta) y+\beta\left(X_{t}-x\right)\right) .
\end{aligned}
$$

Proof. We consider the full process $U_{t}^{\alpha, \beta}=\left(X_{t}^{\alpha, \beta}, W_{t}^{\alpha, \beta}\right)=\left(X_{t}+x+y t+(\alpha / 2)(t-\right.$ $\left.s)^{2}+(\beta / 6)(t-s)^{3}, W_{t}+y+\alpha(t-s)+(\beta / 2)(t-s)^{2}\right)=\left(X_{t}^{\alpha, \beta}, W_{t}^{\alpha, \beta}\right)$. This is a diffusion process satisfying the two-dimensional stochastic differential equation

$$
\begin{gathered}
\mathrm{d} X_{t}^{\alpha, \beta}=W_{t}^{\alpha, \beta} \mathrm{d} t, \\
\mathrm{~d} W_{t}^{\alpha, \beta}=(\alpha+(t-s) \beta) \mathrm{d} t+\mathrm{d} W_{t},
\end{gathered}
$$

to be solved for $t \geq s \geq 0$ with initial conditions $U_{s}^{\alpha, \beta}=(x, y)$.

We want $W_{t}^{\alpha, \beta}$ to be a Brownian motion under a new probability. This is a straightforward application of Girsanov's theorem (or the particular case of Cameron-Martin's formula). We define

$$
L_{t}^{\alpha, \beta}:=-\int_{s}^{t}(\alpha+\beta h) \mathrm{d} W_{h}, \quad 0 \leq s \leq t .
$$

This is a martingale for $\mathcal{F}_{s, t}$ satisfying

$$
\left\langle L^{\alpha, \beta}, L^{\alpha, \beta}\right\rangle_{t}=\int_{s}^{t}(\alpha+\beta h)^{2} \mathrm{~d} h ;
$$

therefore, $\mathrm{E}\left[\exp \left(\frac{1}{2}\left\langle L^{\alpha, \beta}, L^{\alpha, \beta}\right\rangle_{t}\right)\right]<\infty, 0 \leq s \leq t<\infty$. We conclude from Novikov's criterion that $W_{t}-\left\langle W, L^{\alpha, \beta}\right\rangle_{t}=W_{t}^{\alpha, \beta}$ is a Brownian motion under a new probability, noted $\mathrm{P}_{s,(x, y)}^{\alpha, \beta}$.

The Radon-Nikodym derivative $D_{s,(x, y)}^{\alpha, \beta}(t)$ of this new probability with respect to the initial probability $\mathrm{P}_{s,(x, y)}$ is given by Girsanov's theorem and is equal to

$$
\exp \left(L_{t}^{\alpha, \beta}-\frac{1}{2}\left\langle L^{\alpha, \beta}, L^{\alpha, \beta}\right\rangle_{t}\right)
$$


This can be written as

$$
\begin{aligned}
& D_{s,(x, y)}^{\alpha, \beta}(t)=\left.\frac{\mathrm{dP}^{\alpha, \beta}}{\mathrm{dP}}\right|_{\mathcal{F}_{s, t}} \\
&=\exp \left(-\frac{1}{2} \int_{s}^{t}(\alpha+h \beta)^{2} \mathrm{~d} h+\int_{s}^{t}(\alpha+h \beta) \mathrm{d} W_{h}\right) \\
&=\exp \left(-\frac{1}{2}\left(\alpha^{2}(t-s)+\alpha \beta\left(t^{2}-s^{2}\right)+\frac{1}{3} \beta^{2}\left(t^{3}-s^{3}\right)\right)\right. \\
&\left.\quad-\alpha\left(W_{t}-y\right)-\beta\left(t W_{t}-s y\right)+\beta\left(X_{t}-x\right)\right),
\end{aligned}
$$

using Itô's formula for the process $t W_{t}$. Hence, we obtain a formula equivalent to (9).

Remark 2. In the proof of Theorem 1 we have seen that the IWP comes from the stochastic integration of the function $\alpha+\beta t$ with respect to the Brownian density. If we had chosen a polynomial of degree greater than 1 , the integration by parts would have produced higher-order integrals of the Brownian motion that we do not want to deal with since we have no knowledge of their first hitting time. This is the reason why in the sequel we study the first hitting time of the IWP to cubic boundaries and why we cannot go further. This method does not generalize to polynomial boundaries of degree larger than three. However, we show that this is sufficient to approximate the probability distribution of the first hitting time of the IWP and of other DIPs to general curved boundaries, precisely by approximating these boundaries with piecewise-cubic polynomials.

We note that

$$
\begin{gathered}
d^{\alpha, \beta}(s, x, y ; t, u, v)=\exp \left(-\frac{1}{6} \beta^{2}\left(t^{3}-s^{3}\right)-\frac{1}{2} \alpha \beta\left(t^{2}-s^{2}\right)-\frac{1}{2} \alpha^{2}(t-s)\right. \\
-(\alpha+t \beta) v+(\alpha+s \beta) y+\beta(u-x)),
\end{gathered}
$$

the PDF of the new probability with respect to the initial one.

Theorem 2. Let $\tau_{C}$ be the first hitting time of the standard IWP to the cubic curve $C$ whose equation is

$$
C(t-s)=a+b(t-s)+\frac{\alpha}{2}(t-s)^{2}+\frac{\beta}{6}(t-s)^{3}, \quad t \geq s .
$$

Under the reference probability $\mathrm{P}$, the law of the random variable $\left(\tau_{C}, W_{\tau_{C}}\right)$ satisfies the equation

$$
\begin{aligned}
\mathrm{P}_{s,(x, y)}\left[\tau_{C} \in \mathrm{d} t, W_{\tau_{C}} \in \mathrm{d} z\right] \\
=d^{-\alpha,-\beta}\left(s, x, y-b ; t, a, z-b-\alpha(t-s)-\frac{\beta}{2}(t-s)^{2}\right) \\
\quad \times \mathrm{P}_{s,(x, y-b)}\left[\tau_{a} \in \mathrm{d} t, W_{\tau_{a}}+b+\alpha\left(\tau_{a}-s\right)+\frac{\beta}{2}\left(\tau_{a}-s\right)^{2} \in \mathrm{d} z\right] .
\end{aligned}
$$

The second term on the right-hand side is given by Lachal's formula, (8), if a $\neq x$, or by McKean's formula, (7), if $a=x$. 
Proof. Let $\Gamma \subset \mathbb{R}$ be a measurable set, and let $t \geq s \geq 0$. We have, by the change of probability formula,

$$
\begin{aligned}
& \mathrm{P}_{s,(x, y)}^{\alpha, \beta}\left[\tau_{a} \leq t, W_{\tau_{a}} \in \Gamma\right]=\mathrm{E}_{s,(x, y)}\left[\mathbf{1}_{s \leq \tau_{a} \leq t, W_{\tau_{a}} \in \Gamma} D_{s,(x, y)}^{\alpha, \beta}(t)\right] \\
& =\mathrm{E}_{s,(x, y)}\left[\mathbf{1}_{s \leq \tau_{a} \leq t, W_{\tau_{a}} \in \Gamma} \mathrm{E}_{s,(x, y)}\left[D_{s,(x, y)}^{\alpha, \beta}(t) \mid \mathcal{F}_{s \vee \tau_{a}, t \wedge \tau_{a}}\right]\right] \\
& =\mathrm{E}_{s,(x, y)}\left[\mathbf{1}_{s \leq \tau_{a} \leq t, W_{\tau_{a}} \in \Gamma} D_{s,(x, y)}^{\alpha, \beta}\left(t \wedge \tau_{a}\right)\right] \\
& =\mathrm{E}_{s,(x, y)}\left[\mathbf{1}_{s \leq \tau_{a} \leq t, W_{\tau_{a}} \in \Gamma} D_{s,(x, y)}^{\alpha, \beta}\left(\tau_{a}\right)\right] \\
& =\int_{\Gamma} \int_{s}^{t} D_{s,(x, y)}^{\alpha, \beta}\left(t^{\prime}\right) \mathrm{P}_{s,(x, y)}\left[\tau_{a} \in \mathrm{d} t^{\prime} ; W_{\tau_{a}} \in \mathrm{d} z\right] .
\end{aligned}
$$

In going from the second to the third equation we used the fact that, according to Girsanov's theorem, $D_{s,(x, y)}^{\alpha, \beta}(t)$ is a martingale.

If $x \neq a$, the last probability is given by Lachal's formula, (8), and gives the density of the hitting time of $\left(\tau_{a}, W_{\tau_{a}}\right)$ under the new probability $\mathrm{P}_{s,(x, y)}^{\alpha, \beta}$ :

$$
\begin{aligned}
D_{s,(x, y)}^{\alpha, \beta}(t)|z| & \left(p_{t}(x, y ; a, z)\right. \\
& \left.-\int_{0}^{t} \int_{0}^{+\infty} \mathrm{P}_{(0,-|z|)}\left[\tau_{0} \in \mathrm{d} s^{\prime} ; W_{\tau_{0}} \in \mathrm{d} \mu\right] p_{t-s^{\prime}}(x, y ; a,-\varepsilon \mu)\right) \mathbf{1}_{A}(z),
\end{aligned}
$$

where $A=[0, \infty)$ if $x<a$ and $A=(-\infty, 0]$ if $x>a, \varepsilon=\operatorname{sgn}(a-x)$, and $\mathrm{P}_{(0,-|z|)}$ is given by McKean's formula, (7).

We are interested in the probability density under $\mathrm{P}$ of the first hitting time of the curve $C(t-s)$. This hitting time reads

$$
\begin{aligned}
\tau_{C} & =\inf \left\{t>s, X_{t}=C(t-s) \mid X_{s}=x, W_{s}=y\right\} \\
& =\inf \left\{t>s, X_{t}-\frac{\beta}{6}(t-s)^{3}-\frac{\alpha}{2}(t-s)^{2}=a \mid X_{s}=x, W_{s}=y-b\right\} \\
& =\inf \left\{t>s, X_{t}^{-\alpha,-\beta}=a \mid X_{s}^{-\alpha,-\beta}=x, W_{s}^{-\alpha,-\beta}=y-b\right\} .
\end{aligned}
$$

Hence, $\tau_{C}$ under $\mathrm{P}_{s, x, y}$ has the same law as $\tau_{a}$ under $\mathrm{P}_{s, x, y-b}^{-\alpha,-\beta}$. The corresponding location of $W_{\tau_{a}}^{-\alpha,-\beta}$ is $W_{\tau_{a}}+b+\alpha(t-s)+(\beta / 2)(t-s)^{2}$.

So, eventually the law of $\tau_{C}, W_{\tau_{C}}$ under $\mathrm{P}$ reads

$$
\begin{aligned}
\mathrm{P}_{s,(x, y)}\left[\tau_{C} \in \mathrm{d} t, W_{\tau_{C}} \in \mathrm{d} z\right] \\
=d^{-\alpha,-\beta}\left(s, x, y-b ; t, a, z-b-\alpha\left(\tau_{a}-s\right)-\frac{\beta}{2}\left(\tau_{a}-s\right)^{2}\right) \\
\quad \times \mathrm{P}_{s,(x, y-b)}\left[\tau_{a} \in \mathrm{d} t, W_{\tau_{a}}+b+\alpha\left(\tau_{a}-s\right)+\frac{\beta}{2}\left(\tau_{a}-s\right)^{2} \in \mathrm{d} z\right],
\end{aligned}
$$

which is exactly (10). If $x \neq a$, this formula reads

$$
\begin{aligned}
\mathrm{P}_{s,(x, y)}\left[\tau_{C} \in \mathrm{d} t, W_{\tau_{C}} \in \mathrm{d} z\right]= & d^{-\alpha,-\beta}\left(s, x, y-b ; t, a, z-b-\alpha(t-s)-\frac{\beta}{2}(t-s)^{2}\right) \\
& \times l_{x, y-b}^{a}\left(t-s, z-b-\alpha(t-s)-\frac{\beta}{2}(t-s)^{2}\right) \mathrm{d} t \mathrm{~d} z,
\end{aligned}
$$


where $l_{x, y}^{\alpha}$ is Lachal's density, (8). If $x=a$, the same calculus using McKean's formula, (7), gives us the following formula of the hitting time probability density using the same method:

$$
\begin{aligned}
\mathrm{P}_{s,(x, y)}\left[\tau_{C} \in \mathrm{d} t, W_{\tau_{C}} \in \mathrm{d} z\right]= & d^{-\alpha,-\beta}\left(s, x, y-b ; t, a, z-b-\alpha(t-s)-\frac{\beta}{2}(t-s)^{2}\right) \\
& \times m\left(t-s, y-b, z-b-\alpha(t-s)-\frac{\beta}{2}(t-s)^{2}\right) \mathrm{d} t \mathrm{~d} z .
\end{aligned}
$$

\section{Approximation of the first hitting time of the IWP to a general boundary}

In this section we provide the formula of the first hitting time of the IWP to a piecewise-cubic function and use it to compute an approximation formula of the law of the first hitting time of the IWP to a general smooth curved boundary. We also provide the convergence rate of this approximation. This approach inspired the works [2], [23], [25], and [32] on the first hitting time of the Brownian motion to a general boundary. In [25] and [32] the authors used the formula of the first hitting time of the Brownian motion to an affine boundary to derive an approximation of the first hitting time of the Brownian motion to a curved boundary, approximated by a piecewise-affine boundary. The rate of approximation is computed in [2]. This approach is here applied to our problem for the IWP. Here the boundary is approximated by a cubic spline, for which we compute an explicit formula for the PDF of the first hitting time of the IWP. We then compute the convergence speed to the first hitting time of the IWP to a general boundary of the first hitting time of a cubic spline approximation.

\subsection{First hitting time to a continuous piecewise-cubic function}

In this subsection we consider the first hitting time of an IWP to a continuous piecewise-cubic function $C(t)$ defined on the interval $[0, T]$ by

$$
C(t)=\sum_{i=0}^{n-1}\left(a_{i}+b_{i}\left(t-t_{i}\right)+\frac{\alpha_{i}}{2}\left(t-t_{i}\right)^{2}+\frac{\beta_{i}}{6}\left(t-t_{i}\right)^{3}\right) \mathbf{1}_{\left[t_{i}, t_{i+1}\right)}(t) .
$$

The coefficients $\left\{\left(a_{i}, b_{i}, \alpha_{i}, \beta_{i}\right), i=1, \ldots, n\right\}$ are constant on each interval $\left[t_{i}, t_{i+1}\right), i=$ $1, \ldots, n-1$. The continuity assumption requires that

$$
a_{i+1}=a_{i}+b_{i}\left(t_{i+1}-t_{i}\right)+\frac{\alpha_{i}}{2}\left(t_{i+1}-t_{i}\right)^{2}+\frac{\beta_{i}}{6}\left(t_{i+1}-t_{i}\right)^{3} \quad \text { for all } i \in\{1, \ldots, n-1\} .
$$

Remark. This continuity assumption is not essential. Nevertheless we limit ourselves to a continuous boundary because it is sufficient to find good approximations of the first hitting time PDF with continuous functions, since we prove in Theorem 4, below, that, for Lipschitz continuous boundaries, there exists a density for the first hitting time. If the boundary was not continuous then the density function of the first hitting times would have atoms at the points of discontinuity of the boundary. This could be handled at the cost of an unnecessary increase in technical difficulty.

We denote by $\left(U_{t}\right)_{t \geq 0}$ the two-dimensional process $\left(X_{t}, W_{t}\right)_{t \geq 0}$ and assume that the starting point $U_{0}$ is fixed:

$$
X_{0}=x, \quad W_{0}=y .
$$




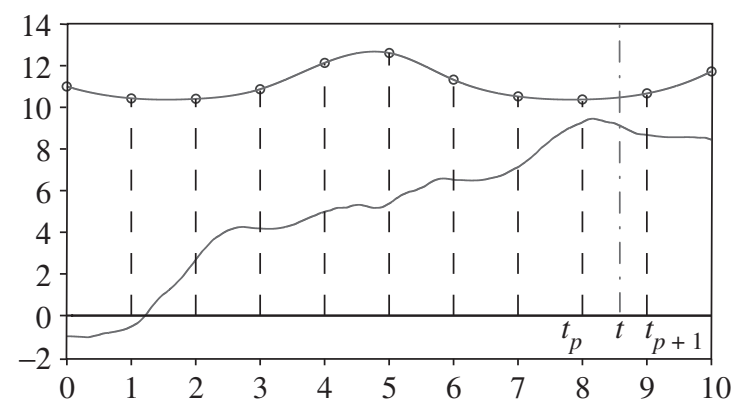

FIGURE 2: Principle of the proof of Theorem 3: the probability that the first hitting time is greater than $t \in\left[t_{p}, t_{p+1}\right)$.

We recall that the process $\left(U_{t}\right)_{t}$ is strongly Markovian with transition measure (6). We denote by $\tau_{C}^{s}$ the first hitting time of the process $\left(X_{t}\right)_{t \geq s}$ to the curve $C$ before the time $T$, i.e.

$$
\tau_{C}^{s}:=\inf \left\{t>s ; X_{t}=C(t)\right\} .
$$

Let us fix $t \in[0, T)$, and denote by $p$ the index of the bin containing $t$ (i.e. $t \in\left[t_{p}, t_{p+1}\right)$ ). The principle of the proof is to use the strong Markov property of $\left(U_{t}\right)_{t}$ to express $\mathrm{P}\left[\tau_{C}^{0} \geq t \mid U_{0}\right]$ recursively as an integral of a product of $p+1$ terms. Of these terms $p$ are related to the results of Subsection 3.2 and their analytical expression is obtained from Theorem 2; see Figure 2.

We know that $\tau_{C}^{s}$ is a stopping time under the filtration associated to $U$, which is strongly Markovian. The event $\left\{U_{t_{1}}=u_{1}, \tau_{C}^{0} \geq t_{1}, U_{0}\right\}$ is in $\mathcal{F}^{U_{t_{1}}}$; therefore, $\mathrm{P}\left[\tau_{C}^{0} \geq t \mid U_{t_{1}}=\right.$ $\left.u_{1}, \tau_{C}^{0} \geq t_{1}, U_{0}\right]=\mathrm{P}\left[\tau_{C}^{t_{1}} \geq t \mid U_{t_{1}}=u_{1}\right]$. It follows that

$$
\begin{aligned}
\mathrm{P}\left[\tau_{C}^{0} \geq t \mid U_{0}\right] & =\int^{(2)} \mathrm{P}\left[\tau_{C}^{0} \geq t \mid U_{t_{1}}=u_{1}, \tau_{C}^{0} \geq t_{1}, U_{0}\right] \mathrm{P}\left[U_{t_{1}} \in \mathrm{d} u_{1}, \tau_{C}^{0} \geq t_{1} \mid U_{0}\right] \\
& =\int^{(2)} \mathrm{P}\left[\tau_{C}^{t_{1}} \geq t \mid U_{t_{1}}=u_{1}\right] \mathrm{P}\left[U_{t_{1}} \in \mathrm{d} u_{1}, \tau_{C}^{0} \geq t_{1} \mid U_{0}\right],
\end{aligned}
$$

where ' $\int(N)$, denotes an integral on $\mathbb{R}^{N}$. The first term in this integral is similar to the left-hand side of the equation. By an immediate recursion we obtain

$$
\begin{aligned}
& \mathrm{P}\left[\tau_{C}^{0} \geq t \mid U_{0}\right]= \int^{(4)} \mathrm{P}\left[\tau_{C}^{t_{2}} \geq t \mid U_{t_{2}}=u_{2}\right] \mathrm{P}\left[U_{t_{2}} \in \mathrm{d} u_{2}, \tau_{C}^{t_{1}} \geq t_{2} \mid U_{t_{1}}=u_{1}\right] \\
& \times \mathrm{P}\left[U_{t_{1}} \in \mathrm{d} u_{1}, \tau_{C}^{0} \geq t_{1} \mid U_{0}\right] \\
&= \\
&=\int^{(2 p)} \mathrm{P}\left[\tau_{C}^{t_{p}} \geq t \mid U_{t_{p}}=u_{p}\right] \mathrm{P}\left[U_{t_{p}} \in \mathrm{d} u_{p}, \tau_{C}^{t_{p-1}} \geq t_{p} \mid U_{t_{p-1}}=u_{p-1}\right] \\
& \quad \times \mathrm{P}\left[U_{t_{p-1}} \in \mathrm{d} u_{p-1}, \tau_{C}^{t_{p-2}} \geq t_{p-1} \mid U_{t_{p-2}}=u_{p-2}\right] \times \cdots \\
& \quad \times \mathrm{P}\left[U_{t_{1}} \in \mathrm{d} u_{1}, \tau_{C}^{0} \geq t_{1} \mid U_{0}\right],
\end{aligned}
$$

Note that the integration variables $\left(u_{i}\right)_{\{i=1, \ldots, p\}}$ are two-dimensional. 
The terms in the product of (14) are of the same kind. Their expression is given by Theorem 3 . Indeed, since

$$
\left\{U_{t_{k}} \in \mathrm{d} u_{k}, \tau_{C}^{t_{k-1}} \geq t_{k}\right\}=\left\{U_{t_{k}} \in \mathrm{d} u_{k}\right\} \backslash\left\{U_{t_{k}} \in \mathrm{d} u_{k}, \tau_{C}^{t_{k-1}}<t_{k}\right\},
$$

where ' $\backslash$ ' is the set difference, we have

$$
\begin{aligned}
& \mathrm{P}\left[U_{t_{k}} \in \mathrm{d} u_{k}, \tau_{C}^{t_{k-1}} \geq t_{k} \mid U_{t_{k-1}}=u_{k-1}\right] \\
&= \mathrm{P}\left[U_{t_{k}} \in \mathrm{d} u_{k} \mid U_{t_{k-1}}=u_{k-1}\right]-\mathrm{P}\left[U_{t_{k}} \in \mathrm{d} u_{k}, \tau_{C}^{t_{k-1}} \leq t_{k} \mid U_{t_{k-1}}=u_{k-1}\right] \\
&= \mathrm{P}\left[U_{t_{k}} \in \mathrm{d} u_{k} \mid U_{t_{k-1}}=u_{k-1}\right]-\int_{t_{k-1}}^{t_{k}} \mathrm{P}\left[U_{t_{k}} \in \mathrm{d} u_{k}, \tau_{C}^{t_{k-1}} \in \mathrm{d} s \mid U_{t_{k-1}}=u_{k-1}\right] \\
&= \mathrm{P}\left[U_{t_{k}} \in \mathrm{d} u_{k} \mid U_{t_{k-1}}=u_{k-1}\right] \\
& \quad-\int_{t_{k-1}}^{t_{k}} \int_{\mathbb{R}} \mathrm{P}\left[U_{t_{k}} \in \mathrm{d} u_{k} \mid \tau_{C}^{t_{k-1}}=s, W_{s}=y, U_{t_{k-1}}=u_{k-1}\right] \\
& \times \mathrm{P}\left[\tau_{C}^{t_{k-1}} \in \mathrm{d} s, W_{s} \in \mathrm{d} y \mid U_{t_{k-1}}=u_{k-1}\right] \\
&=\left(p_{t_{k}-t_{k-1}}\left(u_{k} ; u_{k-1}\right)\right. \\
&\left.\quad-\int_{t_{k-1}}^{t_{k}} \int_{\mathbb{R}} p_{t_{k}-s}\left(u_{k} ; C(s), y\right) \mathrm{P}\left[\tau_{C}^{t_{k-1}} \in \mathrm{d} s, W_{s} \in \mathrm{d} y \mid U_{t_{k-1}}=u_{k-1}\right]\right) \mathrm{d} u_{k},
\end{aligned}
$$

where $p_{t}(x, y ; u, v)$ is the transition density function (6) of the process $U$. The curve $C$ on the interval $\left[t_{k-1}, t_{k}\right)$ is a fixed cubic function. The hitting time of the IWP starting at $u_{k-1}$ to $C$ has a known density computed in Subsection 3.2 and the term we are interested in can be deduced from the expression we derived previously.

Hence, we have proved the following theorem.

Theorem 3. The law of the first hitting time of the IWP to a continuous piecewise-cubic boundary is given by the formula

$$
\begin{aligned}
\mathrm{P}\left[\tau_{C}^{0} \geq t \mid U_{0}\right]=\int^{(2 p)} \mathrm{P}\left[\tau_{C}^{t_{p}} \geq t \mid U_{t_{p}}=u_{p}\right] \\
\times \prod_{k=1}^{p}\left(p_{t_{k}-t_{k-1}}\left(u_{k} ; u_{k-1}\right)\right. \\
\quad-\int_{t_{k-1}}^{t_{k}} \int_{\mathbb{R}} p_{t_{k}-s}\left(u_{k} ; C(s), y\right) \\
\left.\quad \times \mathrm{P}\left[\tau_{C}^{t_{k-1}} \in \mathrm{d} s, W_{s} \in \mathrm{d} y \mid U_{t_{k-1}}\right]\right) \mathrm{d} u_{k} .
\end{aligned}
$$

\subsection{Approximation of the first hitting time to a general boundary}

In this subsection we derive an approximation of the first hitting time before a given time $T$ of the IWP to a general smooth boundary using the results of the previous section.

Let $f: \mathbb{R} \mapsto \mathbb{R}$ be a continuously differentiable function. Also, let $T>0$ and

$$
0=t_{0}<t_{1}<\cdots<t_{n}=T
$$




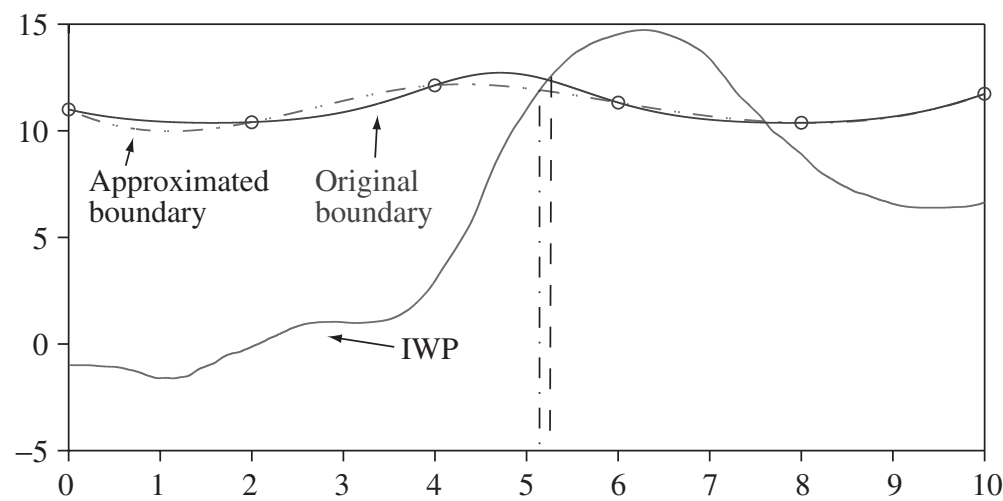

FIGURE 3: Principle of the approximation method: approximating the boundary by a regular piecewisecubic function.

be a partition, noted $\pi$, of the interval $[0, T]$. We denote by $\delta(\pi)$ the mesh step defined as

$$
\delta(\pi)=\max \left\{t_{i+1}-t_{i}, i=0, \ldots, n-1\right\} .
$$

The principle of the method is to approximate the first hitting time of the IWP to the boundary $f$ by the first hitting time of the IWP to a smooth piecewise-cubic function $C_{\pi}$ (see Figure 3 ). The constraints we impose on $C_{\pi}$ are that it can pass through the control points $\left\{\left(t_{i}, f\left(t_{i}\right)\right), i=\right.$ $1, \ldots, n\}$ and to be at least continuously differentiable. There are several ways for defining it; see, e.g. [29]. We assume for simplicity, but it is not essential here, that $f$ is either $C^{2}$ or $C^{4}$. One of the most popular interpolation schemes in the second case is provided by the cubic spline that yields a $C^{2}$ interpolation of $f$ which is an approximation of order four, i.e.

$$
\sup _{t \in[0, T]}\left|f(t)-C_{\pi}(t)\right| \leq K(f) \delta(\pi)^{4},
$$

where $K(f)$ is a function of $f$ only. Therefore, $C_{\pi}(t)$ is given by (13), where the coefficients $a_{i}, b_{i}, \alpha_{i}$, and $\beta_{i}$ are functions of $f$ and provided by the particular interpolation scheme one uses; see, e.g. [29].

We first prove the following theorem.

Theorem 4. The first hitting time of the IWP to a Lipschitz continuous boundary has a density with respect to Lebesgue's measure.

Proof. We assume that $f$ is Lipschitz continuous. Let $L$ denote the Lipschitz continuity constant of $f$. We have

$$
f(s+h)-f(s) \geq-L h \text { for all } 0<s<s+h<t .
$$

For a fixed $t \in[0, T]$, we introduce the boundary $f_{t}(s):=f(t)+L(t-s)$. Now let

$$
\begin{aligned}
\tau_{t} & :=\inf \left\{s>0 ; X_{s}>f_{t}(s) \mid X_{0}=x_{0}, W_{0}=y_{0}\right\} \\
& =\inf \left\{s>0 ; X_{s}+L s>f(t)+L t \mid X_{0}=x_{0}, W_{0}=y_{0}\right\} \\
& =\inf \left\{s>0 ; X_{s}>f(t)+L t \mid X_{0}=x_{0}, W_{0}=y_{0}+L\right\} .
\end{aligned}
$$


Obviously, we have

$$
\mathrm{P}\left[\tau_{f} \in(t, t+h)\right] \leq \mathrm{P}\left[\tau_{t} \in(t, t+h)\right],
$$

and, hence, the stopping time $\tau_{f}$ has a density $p(t)$ with respect to Lebesgue's measure. Moreover, from Lachal's formula we have

$$
p(t) \leq \int_{\mathbb{R}} l_{x_{0}, y_{0}+L}^{f(t)+L t}(t, z) \mathrm{d} z .
$$

We now relate the first hitting time of the cubic approximation $C_{\pi}$ to that of the general boundary $f$.

Theorem 5. The first hitting time of the IWP to the curve $C_{\pi}$ before $T$, converges in law to the first hitting time of the IWP to the curve $f$ before $T$. Furthermore, if $f$ is $C^{2}$ then this convergence is the same order as the approximation of $f$ by the cubic function $C_{\pi}$. More precisely, for a real function $g$, if $P(T, g)$ denotes the probability $\mathrm{P}\left[X_{t} \geq g(t)\right.$ for some $t \in$ $[0, T]]$, there exists a constant $\tilde{K}(f, T)$ that depends on the function $f$ and the time $T$ such that

$$
\left|P\left(T, C_{\pi}\right)-P(T, f)\right| \leq \tilde{K}(f, T)\left\|f-C_{\pi}\right\|_{\infty, T},
$$

where $\|g\|_{\infty, T}=\sup _{s \in[0, T]}|g(s)|$ is the uniform norm on $[0, T]$.

To prove this theorem, we use the following lemma, which gives the density of the random variable $\sup _{s \in[0, t]} X_{s}$.

Lemma 2. Let $t>0$ be a fixed real, and let $S_{t}$ be the random variable defined by

$$
S_{t}=\sup _{s \in[0, t]} X_{s}
$$

Then the law of this random variable is characterized by

$$
\begin{aligned}
& \mathrm{P}_{0, x, y}\left[S_{t} \in \mathrm{d} a\right] \\
& =-\mathrm{d} a \int_{-\infty}^{\infty} \int_{0}^{t}|z|\left(\frac{\partial p_{s}(x, y ; a, z)}{\partial a}-\int_{0}^{s} \int_{0}^{+\infty} \mathrm{P}_{(0,-|z|)}\left[\tau_{0} \in \mathrm{d} u ; W_{\tau_{0}} \in \mathrm{d} \mu\right]\right. \\
& \left.\quad \times \frac{\partial p_{s-u}(x, y ; a,-\varepsilon \mu)}{\partial a}\right) \mathbf{1}_{A}(z) \mathrm{d} z \mathrm{~d} t .
\end{aligned}
$$

Proof. We have

$$
\mathrm{P}_{0, x, y}\left[S_{t} \geq a\right]=\mathrm{P}_{0, x, y}\left[\tau_{a} \leq t\right]=\int_{0}^{t} \int_{-\infty}^{\infty} l_{x, y}^{a}(t, z) \mathrm{d} t \mathrm{~d} z,
$$

where $l_{x, y}^{a}(t, z)$ is Lachal's density. From the expression of this density and its dependency in $a$, we can see that the random variable $S_{t}$ has a density with respect to Lebesgue's measure, and this measure has the form (17) (this formula is obtained using Lebesgue's theorem of derivation under the sum sign). 
Proof of Theorem 5. This result comes directly from the existence of a smooth density of the random variable $S_{t}$, the uniform convergence of the curve $C_{\pi}$ to $f$, and the smoothness of the function $f$. Let $U_{0}$ be the initial conditions, $X_{0}=x$ and $W_{0}=y$, such that $x<f(0)$.

We have

$$
P(T, f+\varepsilon) \leq P(T, f) \leq P(T, f-\varepsilon) .
$$

Assume that $f_{\varepsilon}$ is a uniform approximation of $f$ such that

$$
\left\|f_{\varepsilon}-f\right\|_{\infty, T} \leq \varepsilon
$$

We have

$$
\begin{aligned}
\mid P(T, & \left.f_{\varepsilon}\right)-P(T, f) \mid \\
& \leq P(T, f-\varepsilon)-P(T, f+\varepsilon) \\
& =P(T, f-\varepsilon)-P(T, f)+P(T, f)-P(T, f+\varepsilon) \\
& =\mathrm{P}\left[-\varepsilon \leq \sup _{s \in[0, t]}\left(X_{s}-f(s)\right) \leq 0\right]+\mathrm{P}\left[0 \leq \sup _{s \in[0, t]}\left(X_{s}-f(s)\right) \leq \varepsilon\right] \\
& :=\Delta_{-\varepsilon}(f)+\Delta_{\varepsilon}(f) .
\end{aligned}
$$

We have

$$
\begin{aligned}
\Delta_{\varepsilon}(f)= & \mathrm{P}\left[0 \leq \sup _{s \in[0, t]}\left(X_{s}-f(s)\right) \leq \varepsilon \mid U_{0}\right] \\
= & \int_{0}^{t} \int_{\mathbb{R}} \mathrm{P}\left[\tau_{f} \in \mathrm{d} s, W_{\tau_{f}} \in \mathrm{d} z \mid U_{0}\right] \\
& \times \mathrm{P}\left[\sup _{v \in[s, t]}\left(X_{v}-f(v)\right) \leq \varepsilon \mid X_{s}=f(s), W_{s}=z\right] \\
= & \int_{0}^{t} \int_{f^{\prime}(s)}^{\infty} \mathrm{P}\left[\tau_{f} \in \mathrm{d} s, W_{\tau_{f}} \in \mathrm{d} z \mid U_{0}\right] \\
& \times \mathrm{P}\left[\sup _{v \in[s, t]}\left(X_{v}-f(v)\right) \leq \varepsilon \mid X_{s}=f(s), W_{s}=z\right] .
\end{aligned}
$$

The last equality is a consequence of the fact that $\mathrm{P}\left[W_{\tau_{f}}>f^{\prime}\left(\tau_{f}\right)\right]=1$, i.e. $z \geq f^{\prime}(s)$ almost surely. We can conclude to the convergence of the approximation by virtue of Lebesgue's theorem. Indeed, let $s \in[0, t]$ and $z>f^{\prime}(s)$. Then the process $\left(X_{t}-f(t)\right)_{t \geq s}$ conditioned by $\left\{X_{s}=f(s), W_{s}=z\right\}$ is a differentiable process starting from 0 with a strictly positive derivative at 0 implying that

$$
\mathrm{P}\left[\sup _{v \in[s, t]}\left(X_{v}-f(v)\right) \leq \varepsilon \mid X_{s}=f(s), W_{s}=z\right] \rightarrow 0 \quad \text { as } \varepsilon \rightarrow 0 .
$$

Furthermore, $\mathrm{P}\left[\sup _{v \in[s, t]}\left(X_{v}-f(v)\right) \leq \varepsilon \mid X_{s}=f(s), W_{s}=z\right] \leq 1$, which is integrable under the measure $\mathrm{P}\left[\tau_{f} \in \mathrm{d} s, W_{\tau_{f}} \in \mathrm{d} z \mid U_{0}\right]$, so Lebesgue's theorem applies and we have the following expected result:

$$
\Delta_{\varepsilon}(f) \rightarrow 0 \quad \text { as } \varepsilon \rightarrow 0
$$


The same argument applies for the term $\Delta_{-\varepsilon}(f)$ in (18). Indeed, we can bound this probability for $\varepsilon \leq(f(0)-x) / 2$ by

$$
\begin{aligned}
\Delta_{-\varepsilon}(f) & =\mathrm{P}\left[-\varepsilon \leq \sup _{s \in[0, t]} X_{s}-f(s) \leq 0 \mid X_{0}=x, W_{0}=y\right] \\
& =\mathrm{P}\left[0 \leq \sup _{s \in[0, t]} X_{s}-f(s) \leq \varepsilon \mid X_{0}=x+\varepsilon, W_{0}=y\right] \\
& \leq \mathrm{P}\left[0 \leq \sup _{s \in[0, t]} X_{s}-f(s) \leq \varepsilon \mid X_{0}=\frac{x+f(0)}{2}, W_{0}=y\right] \\
& \rightarrow 0 \quad \text { as } \varepsilon \rightarrow 0 .
\end{aligned}
$$

We obtain a stronger result if the boundary $f$ is $C^{2}$. Lemma 2 ensures that the random variable $\sup _{s \in[0, t]} X_{s}$ has a smooth density with respect to Lebesgue's measure. From Girsanov's theorem, under the probability $\mathrm{Q}$, defined by

$$
\left.\frac{\mathrm{dQ}}{\mathrm{dP}}\right|_{\mathcal{F}_{t}}=\exp \left(\int_{0}^{t} f^{\prime \prime}(s) \mathrm{d} W_{s}-\frac{1}{2} \int_{0}^{t} f^{\prime \prime}(s)^{2} \mathrm{~d} s\right)=: L_{s},
$$

the process $\sup _{v \in[s, t]}\left(X_{v}-f(v)\right)$ has the law of the sup over $[s, t]$ of an IWP, which is given by (17), let us note it $p_{L}(s)$. For $z>f^{\prime}(s)$, the probability that appears at the right-hand side of (19) is equal to

$$
\begin{aligned}
\int_{0}^{\varepsilon} p_{L}(s) \mathrm{E}_{\mathrm{Q}}\left[L_{s}^{-1}\right] \mathrm{d} s & =\int_{0}^{\varepsilon} p_{L}(s) \mathrm{E}_{\mathrm{Q}}\left[\tilde{L}_{s}\right] \exp \left(\int_{0}^{s} f^{\prime \prime}(u)^{2} \mathrm{~d} u\right) \mathrm{d} s \\
& =\int_{0}^{\varepsilon} p_{L}(s) \exp \left(\int_{0}^{s} f^{\prime \prime}(u)^{2} \mathrm{~d} u\right) \mathrm{d} s,
\end{aligned}
$$

since $\tilde{L}_{s}=\exp \left(-\int_{0}^{t} f^{\prime \prime}(s) \mathrm{d} W_{s}-\frac{1}{2} \int_{0}^{t} f^{\prime \prime}(s)^{2} \mathrm{~d} s\right)$ is a martingale. The last integral is $O(\varepsilon)$.

So eventually, again using the same bound as (19), we have the expected result: there exists a constant $\tilde{\tilde{K}}(f, T)$ such that

$$
\left|P\left(T, f_{\varepsilon}\right)-P(T, f)\right| \leq \tilde{\tilde{K}}(f, T) \varepsilon .
$$

Finally, if $f$ is $C^{4}$ and $C_{\pi}$ is a cubic spline interpolation of $f$, we have the convergence estimation (15) which yields

$$
\begin{aligned}
\left|P\left(T, C_{\pi}\right)-P(T, f)\right| & \leq 2 \tilde{\tilde{K}}(f, T)\left\|C_{\pi}-f\right\|_{\infty} \\
& \leq 2 \tilde{K}(f, T) \delta(\pi)^{4} .
\end{aligned}
$$

\section{Approximation of the first hitting time of a general DIP to a general boundary}

In this section we derive an approximation formula for the PDF of the first hitting time of a general DIP to a general smooth boundary. Here again the idea is to use the formulae we obtained in Section 3 to build approximations on a partition of a given time interval $[0, T]$ (see Figure 4).

Thanks to Lemma 1, we can restrict ourselves to the first hitting time of a process $Y$ defined by

$$
Y_{t}=\int_{0}^{t} g(s) W_{s} \mathrm{~d} s
$$

where $g(\cdot)$ is a continuously differentiable function and $W$ is a standard Brownian motion. 


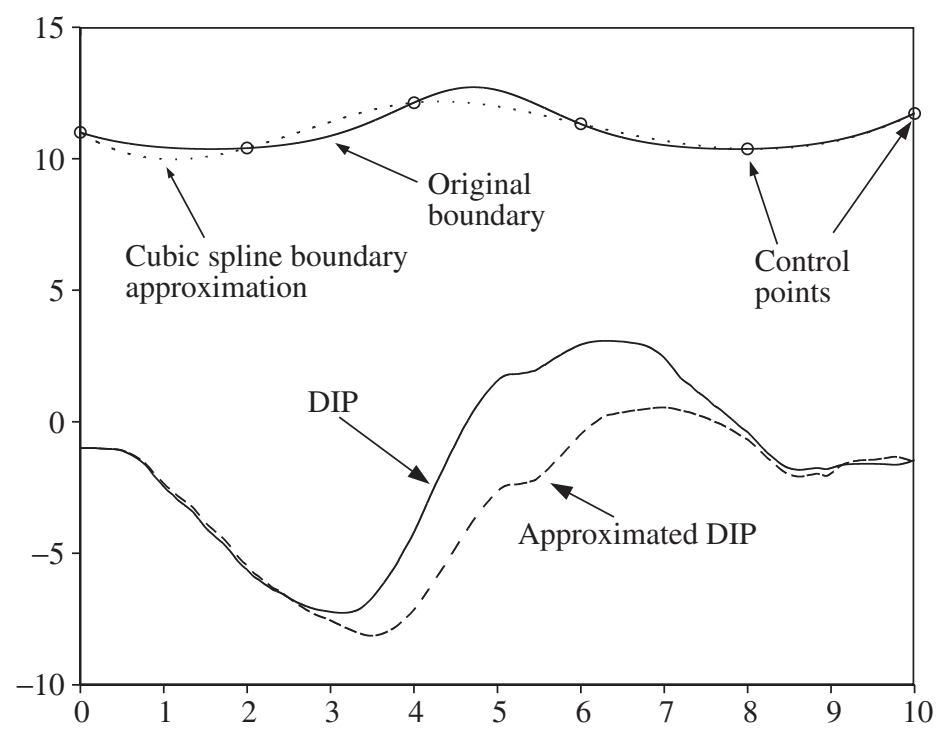

FIGURE 4: Approximation principle for the DIP. The boundary is approximated by asmooth piecewisecubic boundary while the process is approximated by a continuous piecewise IWP.

Let $\pi$ be, as before, a partition of the interval $[0, T]$ with $n$ intervals, i.e.

$$
0=t_{0}<t_{1}<t_{2}<\cdots<t_{n}=T .
$$

We denote by $g^{\pi}$ the piecewise-constant approximation of $g$ defined by

$$
g^{\pi}(t)=\sum_{i=0}^{n-1} g\left(t_{i}\right) \mathbf{1}_{\left[t_{i}, t_{i+1}\right)}(t),
$$

and we denote by $Y^{\pi}$ the associated DIP defined by

$$
Y_{t}^{\pi}=\int_{0}^{t} g^{\pi}(s) W_{s} \mathrm{~d} s .
$$

Proposition 3. The process $Y_{t}^{\pi}$ converges almost surely to the process $Y_{t}$. Furthermore, there exists a real positive process $Z_{t}$ such that

$$
\sup _{0 \leq s \leq t}\left|Y_{s}^{\pi}-Y_{S}\right| \leq \delta(\pi) Z_{t} .
$$

Proof. We have

$$
\begin{aligned}
\sup _{0 \leq s \leq t}\left|Y_{s}^{\pi}-Y_{s}\right| & =\sup _{0 \leq s \leq t}\left|\int_{0}^{s}\left(g^{\pi}(u)-g(u)\right) W_{u} \mathrm{~d} u\right| \\
& \leq \sup _{0 \leq s \leq t} \int_{0}^{s}\left|g^{\pi}(u)-g(u)\right|\left|W_{u}\right| \mathrm{d} u \\
& \leq\left(\sup _{0 \leq s \leq t}\left|g^{\pi}(u)-g(u)\right|\right) \sup _{0 \leq s \leq t} \int_{0}^{s}\left|W_{u}\right| \mathrm{d} u .
\end{aligned}
$$


We assumed that $g$ was continuously differentiable on $\mathbb{R}^{+}$, so it is uniformly Lipschitz on $[0, T]$. We note that

$$
\left\|g^{\prime}\right\|_{\infty, T}=\sup _{0 \leq s \leq T}\left|g^{\prime}(s)\right|,
$$

the uniform Lipschitz constant. We eventually have

$$
\sup _{0 \leq s \leq t}\left|Y_{s}^{\pi}-Y_{s}\right| \leq \delta(\pi)\left\|g^{\prime}\right\|_{\infty, T} \sup _{0 \leq s \leq t} \int_{0}^{s}\left|W_{u}\right| \mathrm{d} u .
$$

The process $Z_{t}:=\sup _{0 \leq s \leq t} \int_{0}^{s}\left|W_{u}\right| \mathrm{d} u$ is almost surely finite; thus, the process $Y^{\pi}$ converges almost surely to $Y$ when $\delta(\pi) \rightarrow 0$ and we have the upper bound in (21).

Now let $f$ be a smooth function (at least two times continuously differentiable), and let $C^{\pi}$ be the approximating function in (13).

Theorem 6. The first hitting time $\tau^{\pi}$ of the process $Y^{\pi}$ to the curve $C^{\pi}$ converges in law to the first hitting time $\tau_{f}$ of the process $Y$ to the curve $f$.

Proof. A sufficient condition for this convergence is the convergence in law of the process $\sup _{s \in[0, t]}\left(Y_{s}^{\pi}-C_{s}^{\pi}\right)$ to the process $\sup _{s \in[0, t]}\left(Y_{s}-f(s)\right)$, and this convergence is a direct consequence of the calculations above. Here we only assume that $f$ is continuously differentiable (the corresponding piecewise-cubic approximation would be, for example, the Hermite cubic approximation; see [29]).

In this case $C_{t}^{\pi}$ converges to $f$ linearly, i.e. there exists a constant $K(f)>0$ depending on $f$ such that

$$
\sup _{t \in[0, T]}\left|f(t)-C_{\pi}(t)\right| \leq K(f) \delta(\pi) .
$$

We have

$$
\begin{aligned}
\sup _{s \in[0, t]}\left(Y_{s}^{\pi}-C_{s}^{\pi}\right) & \leq \sup _{s \in[0, t]}\left(Y_{t}^{\pi}-Y_{t}\right)+\sup _{s \in[0, t]}\left(f(t)-C_{t}^{\pi}\right)+\sup _{s \in[0, t]}\left(Y_{t}-f(t)\right) \\
& \leq \delta(\pi) Z_{t}+K(f) \delta(\pi)+\sup _{s \in[0, t]}\left(Y_{t}-f(t)\right) .
\end{aligned}
$$

Writing the same estimation on $\sup _{s \in[0, t]}\left(Y_{s}-f(s)\right)$ yields

$$
\left|\sup _{s \in[0, t]}\left(Y_{s}^{\pi}-C_{s}^{\pi}\right)-\sup _{s \in[0, t]}\left(Y_{t}-f(t)\right)\right| \leq \delta(\pi)\left(Z_{t}+K(f)\right) .
$$

Hence, we have the following expected result:

$$
\begin{aligned}
\mathrm{P}\left[\tau^{\pi} \leq t\right] & =\mathrm{P}\left[\sup _{s \in[0, t]}\left(Y_{s}^{\pi}-C_{s}^{\pi}\right)>0\right] \\
& \rightarrow \mathrm{P}\left[\sup _{s \in[0, t]}\left(Y_{s}-f(s)\right)>0\right] \quad \text { as } \delta(\pi) \rightarrow 0,
\end{aligned}
$$

which is equal to $\mathrm{P}\left[\tau_{f} \leq t\right]$.

We now compute the formula of the PDF of the first crossing time of $Y^{\pi}$ to the curve $C^{\pi}$, as we did in Section 4.

We consider $\pi$ to be a fixed partition of the interval $[0, T]$. We denote by $\tau_{\pi}^{s}$ the first hitting time after time $s$ of the process $Y^{\pi}$ to the curve $C^{\pi}$ and we denote by $U^{\pi}$ the two-dimensional 
process $\left(Y_{t}^{\pi}, W_{t}\right)$. The same proof as in Section 4 gives us the following analogous formula to (14):

$$
\begin{aligned}
\mathrm{P}\left[\tau_{\pi}^{0} \geq t \mid U_{0}^{\pi}\right]=\int^{(2 p)} & \mathrm{P}\left[\tau_{\pi}^{t_{p}} \geq t \mid U_{t_{p}}^{\pi}=u_{p}\right] \\
& \times \mathrm{P}\left[\left(U_{t_{p}}^{\pi} \in \mathrm{d} u_{p}, \tau_{\pi}^{t_{p-1}} \geq t_{p} \mid U_{t_{p-1}}^{\pi}=u_{p-1}\right]\right. \\
& \times \mathrm{P}\left[U_{t_{p-1}}^{\pi} \in \mathrm{d} u_{p-1}, \tau_{\pi}^{t_{p-2}} \geq t_{p-1} \mid U_{t_{p-2}}^{\pi}=u_{p-2}\right] \times \cdots \\
& \times \mathrm{P}\left[U_{t_{1}}^{\pi} \in \mathrm{d} u_{1} \mid U_{0}^{\pi}\right] .
\end{aligned}
$$

Here again the terms in the recursion formula are of the same kind, and the only quantities we need to calculate are the conditional probabilities $\mathrm{P}\left[U_{t_{k}}^{\pi} \in \mathrm{d} u_{k}, \tau_{\pi}^{t_{k-1}} \geq t_{k} \mid U_{t_{k-1}}^{\pi}=u_{k-1}\right]$ for $k=1, \ldots, p$.

Note that in the interval $\left[t_{k}, t_{k+1}\right)$ the process $Y_{t}^{\pi}$ reads

$$
Y_{t}^{\pi}=Y_{t_{i}}^{\pi}+g\left(t_{k}\right)\left(X_{t}-X_{t_{k}}\right)
$$

and that $\left(X_{t}-X_{t_{k}}\right)_{t \geq t_{k}}$ is simply an IWP starting from 0 . We then have

$$
\begin{aligned}
& \mathrm{P}\left[U_{t_{k}}^{\pi} \in \mathrm{d} u_{k}, \tau_{\pi}^{t_{k-1}} \geq t_{k} \mid U_{t_{k-1}}^{\pi}=u_{k-1}\right] \\
&= \mathrm{P}\left[U_{t_{k}}^{\pi} \in \mathrm{d} u_{k} \mid U_{t_{k-1}}^{\pi}=u_{k-1}\right]-\mathrm{P}\left[U_{t_{k}}^{\pi} \in \mathrm{d} u_{k}, \tau_{\pi}^{t_{k-1}} \leq t_{p-1} \mid U_{t_{k-1}}^{\pi}=u_{k-1}\right] \\
&= \mathrm{P}\left[U_{t_{k}}^{\pi} \in \mathrm{d} u_{k} \mid U_{t_{k-1}}^{\pi}=u_{k-1}\right] \\
& \quad-\int_{t_{k-1}}^{t_{k}} \mathrm{P}\left[U_{t_{k}}^{\pi} \in \mathrm{d} u_{k}, \tau_{\pi}^{t_{k-1}} \in \mathrm{d} s \mid U_{t_{k-1}}^{\pi}=u_{k-1}\right] \mathrm{P}\left[U_{t_{k}}^{\pi}=d u_{k} \mid U_{t_{k-1}}^{\pi}=u_{k-1}\right] \\
& \quad-\int_{t_{k-1}}^{t_{k}} \int_{\mathbb{R}} \mathrm{P}\left[U_{t_{k}}^{\pi} \in \mathrm{d} u_{k} \mid \tau_{\pi}^{t_{k-1}}=s, W_{s}=y, U_{t_{k-1}}^{\pi}=u_{k-1}\right] \\
& \quad \times \mathrm{P}\left[\tau_{\pi}^{t_{k-1}} \in \mathrm{d} s, W_{s} \in \mathrm{d} y \mid U_{t_{k-1}}^{\pi}=u_{k-1}\right] \\
&=\mathrm{d} u_{k}\left(\tilde{p}\left(t_{k}, u_{k} ; t_{k-1}, u_{k-1}\right)-\int_{t_{k-1}}^{t_{k}} \int_{\mathbb{R}} \tilde{p}\left(t_{k}, u_{k} ; s,\left(C^{\pi}(s), y\right)\right)\right. \\
&\left.\quad \times \mathrm{P}\left[\tau_{\pi}^{t_{k-1}} \in \mathrm{d} s, W_{s} \in \mathrm{d} y \mid U_{t_{k-1}}^{\pi}=u_{k-1}\right]\right)
\end{aligned}
$$

where $\tilde{p}(t, x, y ; s, u, v)$ is the transition function of the process $U^{\pi}$ (for $t \geq s$ ). This function can be deduced from (6) and (22) for $s$ and $t$ in the same bin $\left[t_{k}, t_{k+1}\right)$ and reads

$$
\begin{aligned}
\tilde{p}(t, x, y ; s, u, v) & =\mathrm{P}\left[Y_{t}^{\pi}=x, W_{t}=y \mid Y_{s}^{\pi}=u, W_{s}=v\right] \\
& =\mathrm{P}\left[Y_{s}^{\pi}+g\left(t_{k}\right)\left(X_{t}-X_{s}\right)=x, W_{t}=y \mid Y_{s}^{\pi}=u, W_{s}=v\right] \\
& =\mathrm{P}\left[X_{t}-X_{s}=\frac{x-u}{g\left(t_{k}\right)}, W_{t}=y \mid Y_{s}^{\pi}=u, W_{s}=v\right] \\
& =\mathrm{P}\left[X_{t}-X_{s}=\frac{x-u}{g\left(t_{k}\right)}, W_{t}-W_{s}=y-v \mid Y_{s}^{\pi}=u, W_{s}=v\right] .
\end{aligned}
$$


We have seen in a remark in Subsection 2.2 that, conditionally to $W_{s}$, the increments of the two-dimensional process are independent of $\mathcal{F}_{S}$, so we have

$$
\begin{aligned}
\tilde{p}(t, x, y ; s, u, v) & =\mathrm{P}\left[X_{t-s}=\frac{x-u}{g\left(t_{k}\right)}, W_{t-s}=y-v \mid X_{0}=0, W_{0}=0\right] \\
& =p_{t-s}\left(\frac{x-u}{g\left(t_{k}\right)}, y-v ; 0,0\right) .
\end{aligned}
$$

Hence, the general term (23) of the expansion reads

$$
\begin{aligned}
\mathrm{d} x_{k} \mathrm{~d} y_{k}\left(p_{t_{k}-t_{k-1}}\left(\frac{x_{k}-x_{k-1}}{g\left(t_{k-1}\right)}, y_{k}-y_{k-1} ; 0,0\right)\right. \\
-\int_{t_{k-1}}^{t_{k}} \int_{\mathbb{R}} p_{t_{k}-s}\left(\frac{x_{k}-C^{\pi}(s)}{g\left(t_{k-1}\right)}, y_{k}-y ; 0,0\right) \\
\left.\quad \times \mathrm{P}\left[\tau^{\pi} \in \mathrm{d} s, W_{s} \in \mathrm{d} y \mid U_{t_{k-1}}^{\pi}=u_{k-1}\right]\right) .
\end{aligned}
$$

The last thing to compute is $\mathrm{P}\left[\tau^{\pi} \in \mathrm{d} s, W_{s} \in \mathrm{d} y \mid U_{t_{k-1}}^{\pi}=u_{k-1}\right]$, the law of the hitting time $\tau^{\pi}$ in a given bin $\left[t_{k}, t_{k-1}\right.$ ), which appears in (23). This law can be deduced from that of the first hitting time of the IWP using (22).

Indeed, to compute this probability, we use the fact that, conditionally on the event $\left\{U_{t_{k-1}}^{\pi}=\right.$ $\left.u_{k-1}, t \geq t_{k-1}\right\}$, we have

$$
\begin{aligned}
\tau^{\pi} & =\inf \left\{s>t_{k-1}, Y_{s}^{\pi}=C^{\pi}(s) \mid U_{t_{k-1}}^{\pi}=u_{k-1}\right\} \\
& =\inf \left\{s>t_{k-1}, Y_{t_{k-1}}^{\pi}+g\left(t_{k-1}\right)\left(X_{s}-X_{t_{k-1}}\right)=C^{\pi}(s) \mid U_{t_{k-1}}^{\pi}=u_{k-1}\right\} \\
& =\inf \left\{s>t_{k-1}, X_{s}-X_{t_{k-1}}=\frac{C^{\pi}(s)-Y_{t_{k-1}}^{\pi}}{g\left(t_{k-1}\right)} \mid U_{t_{k-1}}^{\pi}=u_{k-1}\right\} \\
& =\inf \left\{s>0, \tilde{X}_{s}=\frac{C^{\pi}(s)-Y_{t_{k-1}}^{\pi}}{g\left(t_{k-1}\right)} \mid \tilde{X}_{0}=0, \tilde{W}_{0}=u_{t_{k-1}, 2}\right\},
\end{aligned}
$$

where $\left(\tilde{X}_{t}, \tilde{W}_{t}\right)_{t}$ is a standard IWP and $u_{t_{k-1}, 2}$ is the second component of $U_{t_{k-1}}^{\pi}$. Hence, we have

$$
\mathrm{P}\left[\tau^{\pi} \in \mathrm{d} s, W_{s} \in \mathrm{d} y \mid U_{t_{k-1}}^{\pi}=u_{k-1}\right]=\mathrm{P}_{s,\left(0, u_{t_{k-1}, 2}\right)}\left[\tau_{\left(C-Y_{t_{k-1}}^{\pi}\right) / g\left(t_{k-1}\right)} \in \mathrm{d} s, W_{s} \in \mathrm{d} y\right],
$$

and the last expression is given by (11) and (12).

We have proved the following theorem.

Theorem 7. Let $g$ be a Lipschitz continuous real function, let $T>0$, and $\pi$ is the partition of the interval $[0, T]$ :

$$
0=t_{0}<t_{1}<\cdots<t_{n}=T
$$

Let $f$ be a continuously differentiable function. The first hitting time $\tau^{\pi}$ of the approximated process $Y^{\pi}$ defined by (20) to a cubic spline approximation of $f$ on the partition $\pi$, denoted by 
$f^{\pi}$, satisfies the equation

$$
\begin{aligned}
& \mathrm{P}\left[\tau^{\pi} \geq T \mid U_{0}\right] \\
& =\int^{(2 n)} \prod_{k=1}^{n}\left(p_{t_{k}-t_{k-1}}\left(\frac{x_{k}-x_{k-1}}{g\left(t_{k-1}\right)}, y_{k}-y_{k-1} ; 0,0\right)\right. \\
& \quad-\int_{t_{k}-1}^{t_{k}} \int_{\mathbb{R}} p_{t_{k}-s}\left(\frac{x_{k}-f^{\pi}(s)}{g\left(t_{k-1}\right)}, y_{k}-y ; 0,0\right) \\
& \left.\quad \times \mathrm{P}_{s,(0, y)}\left[\tau_{\left(C-x_{k-1}\right) / g\left(t_{k-1}\right)} \in \mathrm{d} s, W_{s} \in \mathrm{d} y\right]\right) \mathrm{d} x_{k} \mathrm{~d} y_{k},
\end{aligned}
$$

where $\mathrm{P}\left[\tau_{C} \in \mathrm{d} s, W_{s} \in \mathrm{d} y\right]$ is given by (11) or (12).

\section{Numerical evaluation}

\subsection{Algorithm}

In this section we propose an algorithm to evaluate the approximation formula we derived in the previous section. The expressions we found for the first hitting time involve integrals on $\mathbb{R}^{2 n}$ when there are $n+1$ points in the mesh, which have no closed-form expression. Numerical computation of these integrals can be quite intricate and time consuming, so a numerical approximation is needed and another approximation is made besides (16) and Theorem 6 . The principle of the numerical approximation we propose is to express this integral as an expectation over a certain probability measure and to use a Monte Carlo algorithm to compute this expectation. The accuracy of this approximation can be assessed through standard procedures for Monte Carlo simulations [20], [28].

Corollary 1. (Of Theorem 3.) Let $\left(X_{t}, W_{t}\right)_{t \geq 0}$ be a standard IWP-Brownian motion pair, and let $f$ be a smooth boundary function. The law of the first hitting time $\tau$ of $X$ to $f$ satisfies, for $t \in\left[t_{p-1}, t_{p}\right)$,

$$
\mathrm{P}\left[\tau_{C} \geq t \mid U_{0}\right]=\mathrm{E}\left[h_{p}\left(\pi, t, X_{t_{1}}, W_{t_{1}}, \ldots, X_{t_{p-1}}, W_{t_{p-1}}, X_{t}, W_{t}\right) \mid U_{0}\right],
$$

where the function $h_{p}$ is defined, for $t \in\left[t_{p-1}, t_{p}\right)$, by

$$
\begin{aligned}
& h_{p}\left(\pi, t, x_{1}, y_{1}, \ldots, x, y\right) \\
& :=\prod_{k=1}^{p-1}\left(1-\int_{t_{k-1}}^{t_{k}} \int_{\mathbb{R}} \frac{p_{t_{k}-s}\left(x_{k}, y_{k}, C(s), z\right)}{p_{t_{k}-t_{k-1}}\left(x_{k}, y_{k}, x_{k-1}, y_{k-1}\right)}\right. \\
& \left.\quad \times \mathrm{P}\left[\tau_{C} \in \mathrm{d} s, W_{\tau_{C}} \in \mathrm{d} z \mid X_{t_{k-1}}=x_{k-1}, W_{t_{k-1}}=y_{k-1}\right]\right) \\
& \quad \times\left(1-\int_{t_{p-1}}^{t} \int_{\mathbb{R}} \frac{p_{t_{p-1}-s}(x, y, C(s), z)}{p_{t-t_{p-1}}\left(x, y, x_{p-1}, y_{p-1}\right)}\right. \\
& \left.\quad \times \mathrm{P}\left[\tau_{C} \in \mathrm{d} s, W_{\tau_{C}} \in \mathrm{d} z \mid X_{t_{p-1}}=x_{p-1}, W_{t_{p-1}}=y_{p-1}\right]\right) .
\end{aligned}
$$

The same method can be applied for the DIP for a given function $g$. 
Corollary 2. (Of Theorem 7.) Let g be a Lipschitz continuous real function, let $\left(X_{t}, W_{t}\right)_{t \geq 0}$ be a standard IWP-Brownian motion pair, let $T>0$, and $\pi$ is the partition of the interval $[0, T]$ :

$$
0=t_{0}<t_{1}<\cdots<t_{n}=T \text {. }
$$

Let $f$ be a continuously differentiable function. The first hitting time $\tau^{\pi}$ of the approximated process $Y^{\pi}$ defined by (20) to a cubic spline approximation of $f$ on the partition $\pi$, denoted $f^{\pi}$, can be computed as the expectation, i.e.

$$
\mathrm{P}\left[\tau^{\pi} \geq t \mid U_{0}\right]=\mathrm{E}\left[h_{p}^{g, \pi}\left(t, X_{t_{1}}, W t_{1}, \ldots, X_{t}, W_{t}\right) \mid U_{0}\right],
$$

where the function $h_{p}^{g, \pi}$ is defined by

$$
\begin{aligned}
& h_{p}^{g, \pi}\left(x_{1}, y_{1} \ldots, x, y\right) \\
&:= \prod_{k=1}^{p-1}\left(\frac{p_{t_{k}-t_{k-1}}\left(\left(x_{k}-x_{k-1}\right) / g\left(t_{k-1}\right), y_{k}-y_{k-1} ; 0,0\right)}{p_{t_{k}-t_{k-1}}\left(x_{k}, y_{k}, x_{k-1}, y_{k-1}\right)}\right. \\
&-\int_{t_{k-1}}^{t_{k}} \int_{\mathbb{R}} \frac{p_{t_{k}-s}\left(\left(x_{k}-f^{\pi}(s)\right) / g\left(t_{k-1}\right), y_{k}-z ; 0,0\right)}{p_{t_{k}-t_{k-1}}\left(x_{k}, y_{k}, x_{k-1}, y_{k-1}\right)} \\
& \times\left(\frac{p_{t-t_{p-1},\left(0, y_{s}\right)}\left(\tau_{\left(C-x_{k-1}\right) / g\left(t_{k-1}\right)}\left(\left(x-x_{p-1}\right) / g\left(t_{p-1}\right), y-y_{p-1} ; 0,0\right)\right.}{p_{t-t_{p-1}}\left(x, y, x_{p-1}, y_{p-1}\right)}\right. \\
&-\int_{t_{p-1}}^{t} \frac{p_{t-s}\left(\left(x-f^{\pi}(s)\right) / g\left(t_{p-1}\right), y-z ; 0,0\right)}{p_{t-t_{p-1}}\left(x, y, x_{p-1}, y_{p-1}\right)} \\
&\left.\times \mathrm{P}_{s,(0, z)}\left[\tau_{\left(C-x_{p-1}\right) / g\left(t_{p-1}\right)} \in \mathrm{d} s, W_{s} \in \mathrm{d} z\right]\right),
\end{aligned}
$$

where $\mathrm{P}\left[\tau_{C} \in \mathrm{d} s, W_{s} \in \mathrm{d} y_{s}\right]$ is given by (11) or (12).

Hence, the problem is now reduced to the computation of the expectation of a certain function of the Gaussian random vector $\left(X_{0}, W_{0}, X_{t_{1}}, W_{t_{1}}, \ldots, X_{t_{n}}, W_{t_{n}}\right)$. This vector is Gaussian of mean 0 and covariance matrix defined by blocks as

$$
K\left(t_{1}, \ldots, t_{n}\right)=\left(\begin{array}{cc}
\frac{t_{j}^{2}}{6}\left(3 t_{i}-t_{j}\right) & \frac{t_{j}}{2}\left(2 t_{i}-t_{j}\right) \\
\frac{t_{j}^{2}}{2} & t_{j}
\end{array}\right)_{(i, j) \in\{0, \ldots, n\}, j \leq i}
$$

The Monte Carlo algorithm we use to compute the expected probability is the following.

(i) Compute the square root $K\left(t_{1}, \ldots, t_{n}\right)^{1 / 2}$ of the covariance matrix (25) (using, for instance, a Cholesky decomposition).

(ii) Generate an independent and identically distributed sample $u=\left[u_{1}, u_{2}, \ldots, u_{2 n}\right]^{\top}$ from the normal standard distribution $\mathcal{N}(0,1)$.

(iii) Compute the transformation $x=K\left(t_{1}, \ldots, t_{n}\right)^{1 / 2} u$.

(iv) Calculate $h_{n}(x)$ or $h_{n}^{g, \pi}(x)$. 
(v) Repeat steps (ii)-(iv) $N$ times and calculate the frequency

$$
P_{N}=\frac{1}{N} \sum_{\text {over the realizations }} h_{n}(x) \text { or } P_{N}=\frac{1}{N} \sum_{\text {over the realizations }} h_{n}^{g, \pi}(x) .
$$

The probability $\mathrm{P}[\tau \geq T]$ is then estimated by $P_{N}$. The standard error of this estimator is given by

\subsection{Numerical results}

$$
\mathscr{E}(N)=\sqrt{\frac{\sum\left(h(x)-P_{N}\right)^{2}}{N(N-1)}} .
$$

Lachal's formula has been implemented using the Gauss integration method. This method is very useful for computing the double integral of Lachal's formula. It allows us to control the precision of the approximation using standard methods (see, e.g. [29]). With this method, we obtain the two-dimensional joint PDF of $\left(\tau_{a}, W_{\tau_{a}}\right)$ conditioned on the starting point. Figure 5 represents the dependence of this law on the starting point. Note that the probability that the hitting
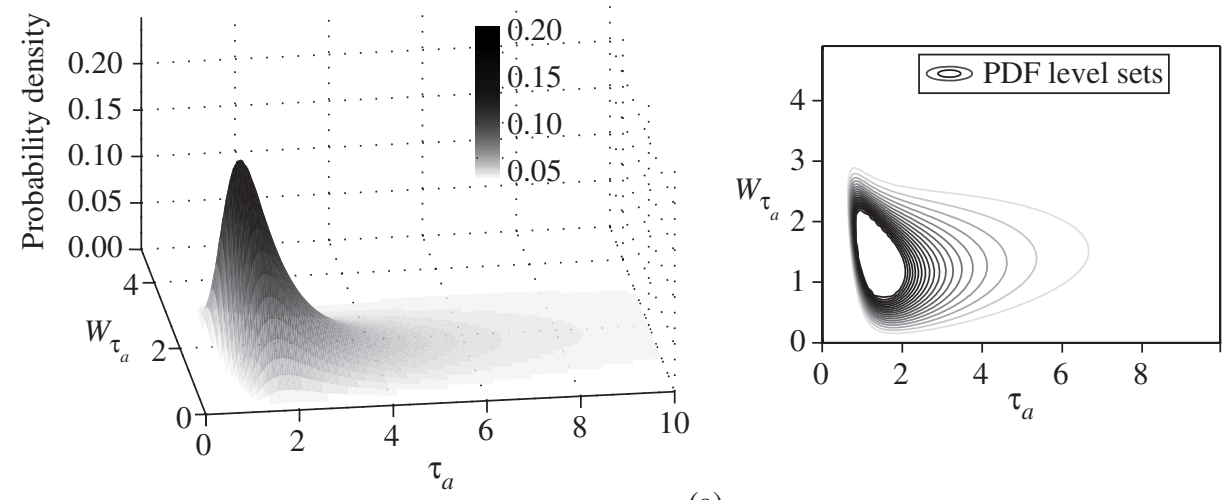

(a)
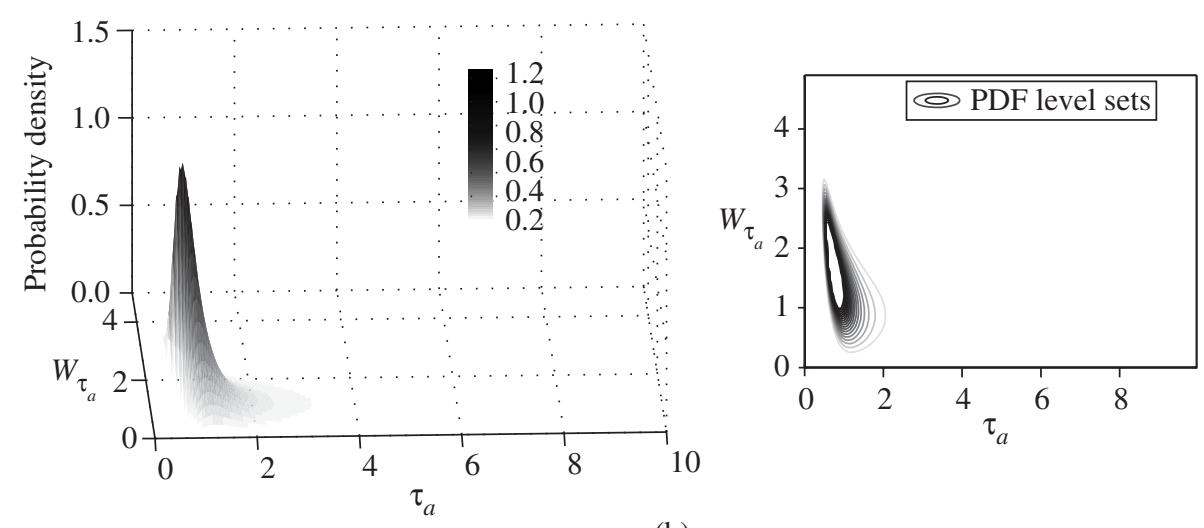

(b)

FIGURE 5: Lachal's PDF for of the first hitting time of the IWP to the constant $a=1$ for initial conditions (a) $X_{0}=0$ and $W_{0}=0$, and (b) $X_{0}=0$ and $W_{0}=1$. Level sets show how the PDF is distributed. The uppermost half level sets are not displayed for the sake of visibility. 


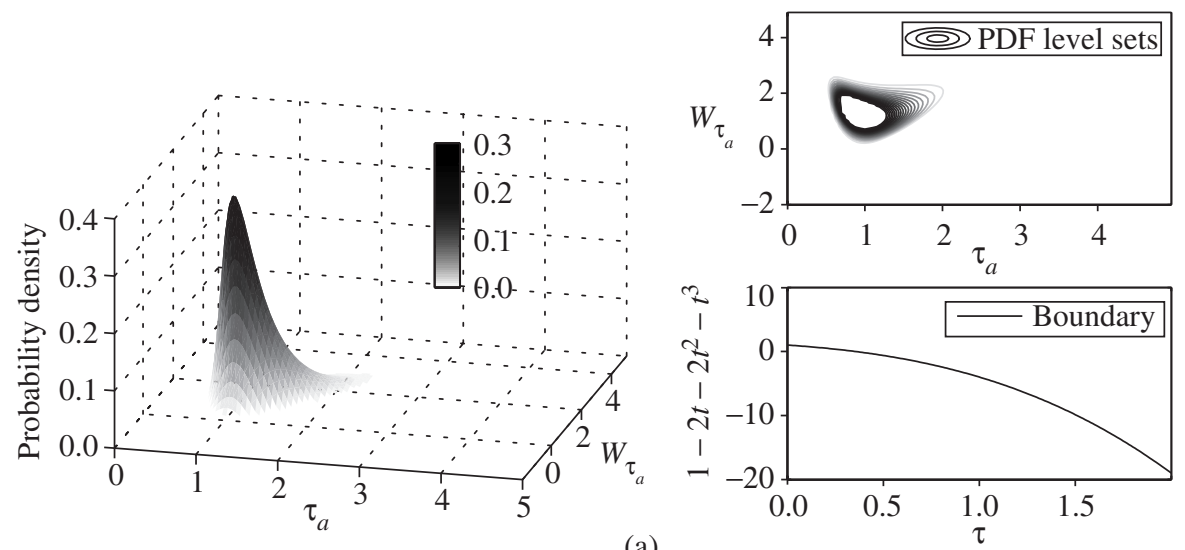

(a)
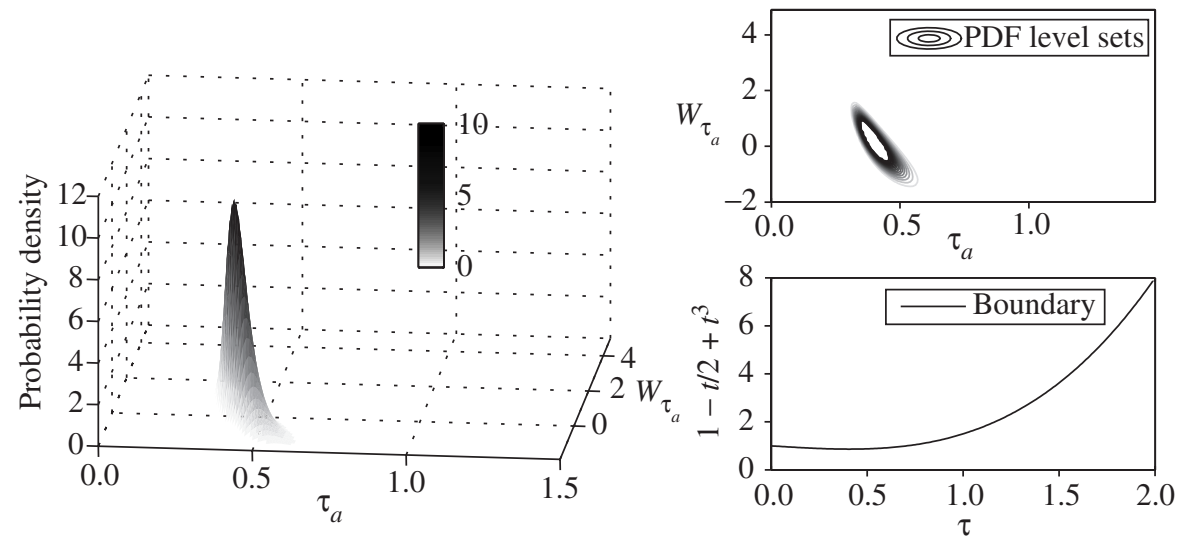

(b)

Figure 6: PDF of the first hitting time of the IWP to the cubic curve (a) $t \mapsto 1-2 t-2 t^{2}-t^{3}$ and (b) $t \mapsto 1-\frac{1}{2} t+t^{3}$, with the initial condition $X_{0}=0$ and $W_{0}=0$. For case (a), the total mass is 1 and, for case (b), the total mass is approximately 0.2578 .

time $\tau_{a}$ is strictly less than $\infty$ is always 1 , as proved in [18]. The process almost surely crosses any given constant. Computation times for a precision set to $10^{-6}$ are around $0.1 \mathrm{~ms}$.

The formula we obtained for a cubic density is simply a transformation of Lachal's density using formulae (11) and (12). It is clear that now the crossing probability will not always be equal to 1 , for instance, when $\beta>0$. Figure 6 illustrates this property and we give a numerical estimation of the probability of crossing the boundary. This probability can be computed using the formulae we obtained, but it is not in the scope of the present paper.

Finally, our last formula can be implemented using different Monte Carlo algorithms. Here we obtain only the inverse cumulative distribution function of the first hitting time. Computation times are quite large, but the main interest of this technique is not computational. In Figure 7 we present some results obtained for the sinusoidal functions $f(x)=\sin (x)$ and $g(x)=\cos (x)$ and a comparison between the results obtained using (24) and a direct integration of Lachal's formula in the case where $f$ and $g$ are identically equal to 1 and the constant boundary is equal 


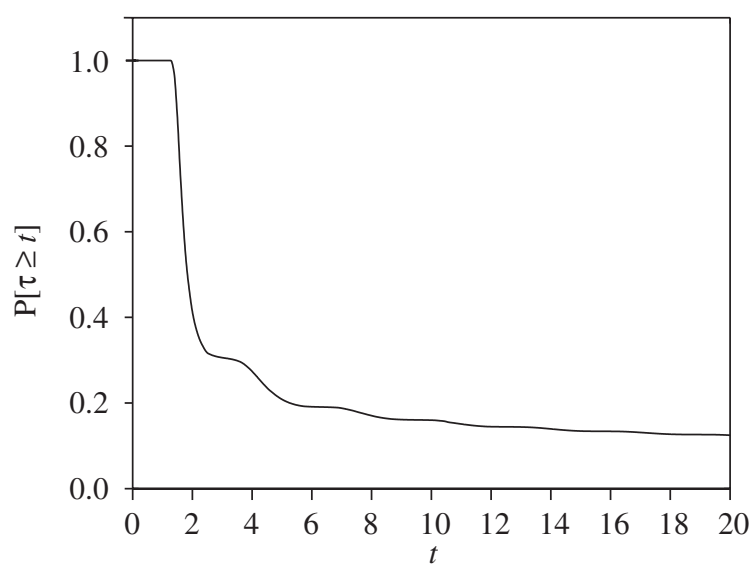

(a)

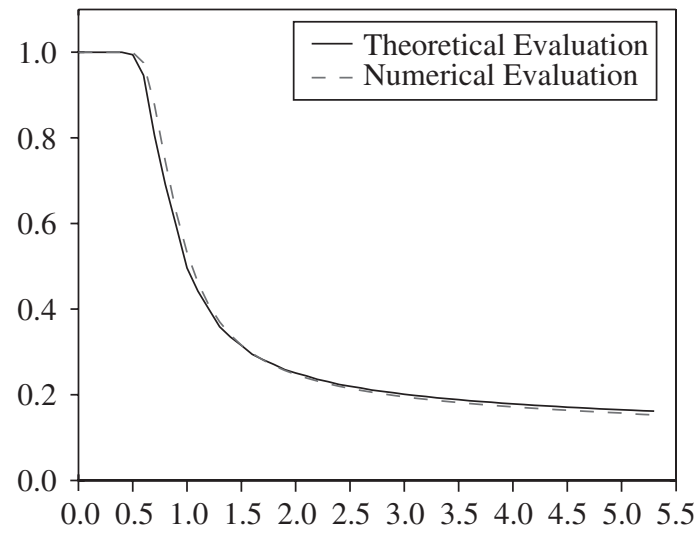

(b)

FIGURE 7: (a) Inverse cumulative distribution of the first hitting time of a linear curve of a DIP defined by the sinusoidal functions $f(x)=\sin (x)$ and $g(x)=\cos (x)$. (b) Inverse cumulative distribution function of the IWP to a constant boundary equal to 1 obtained by our method compared with the theoretical evaluation.

to 1 . In these cases, computation times are reasonable since there is no need to have a large number of points in our partition.

In Table 1 we present results obtained with (24) for the first hitting time of the IWP to the sinusoidal function $t \mapsto 1-\sin (2 t / \pi)$ in the interval $[0,1]$. We discretized the interval with five points (hence, the approximation is of order $10^{-3}$ ). Monte Carlo calculations take quite long to obtain the same order of approximation $\left(10^{6}\right.$ trials for points in $\mathbb{R}^{10}$ per point of the curve). Indeed, the computation of the function inside the integral on $\mathbb{R}^{2 p}$ involves the computation of a fourth-order integral that we compute using a Gauss method of the same order of approximation, which requires a long computation time. Hence, we only show a table of results.

Probably other algorithms would be more efficient, but the corresponding computational issues are outside the scope of the present paper. 
TABLE 1: Table of the probabilities of crossing the sinusoidal function $t \mapsto 1-\sin (2 t / \pi)$ after time $t$ by

\begin{tabular}{cc}
\multicolumn{3}{c}{ an IWP starting at the origin } \\
\hline Time & $\mathrm{P}\left[\tau_{\pi} \geq t \mid U_{0}\right]$ \\
\hline 0.1 & 1.008819 \\
0.2 & 1.003199 \\
0.3 & 1.000000 \\
0.4 & 0.999871 \\
0.5 & 0.999576 \\
0.6 & 0.996399 \\
0.7 & 0.984581 \\
\hline
\end{tabular}

\section{Conclusion}

In this paper we have provided a method of approximation of the probability distribution of the first hitting time of a DIP to a curved boundary. To our knowledge, this is the first result for this problem.

We first obtained a closed-form expression of the probability distribution of the first hitting time of the IWP to a continuous piecewise-cubic boundary.

By approximating a general smooth boundary with a piecewise-cubic function we used this expression to compute an approximation of the probability distribution of the first hitting time of the IWP to any smooth curved boundary, and proved that it converges (very fast in many cases) towards the probability distribution of the first hitting time of the IWP to the original curved boundary.

We then extended the method to solve the problem of approximating the probability distribution of the first hitting time of a DIP to a smooth curved boundary.

Lastly, we sketched a numerical procedure based on a Monte Carlo simulation to compute the probability distribution efficiently.

These results have potential applications in many fields of physics and biology.

\section{Acknowledgements}

This work was partially supported by the 'Fondation d'Entreprise EADS' and the EC IP project \#015879, FACETS.

\section{References}

[1] Anderssen, R. S., Hood, F. R. D. And Weiss, R. (1973). On the numerical solution of Brownian motion processes. J. Appl. Prob. 10, 409-418.

[2] Borovkov, K. And Novikov, A. (2005). Explicit bounds for approximation rates for boundary crossing probabilities for the Wiener process. J. Appl. Prob. 42, 82-92.

[3] Buonocore, A., Nobile, A. G. and Ricciardi, L. M. (1987). A new integral equation for the evaluation of first-passage-time probability densities. Adv. Appl. Prob. 19, 784-800.

[4] Daniels, H. E. (1996). Approximating the first crossing-time density for a curved boundary. Bernoulli 2 , 133-143.

[5] Durbin, J. (1985). The first-passage-density of a continuous Gaussian process to a general boundary. J. Appl. Prob. 22, 99-122. (Correction: 25 (1988), 840.)

[6] Durbin, J. (1992). The first-passage density of the Brownian motion process to a curved boundary. J. Appl. Prob. 29, 291-304.

[7] Favella, L., Reineri, M., Ricciardi, L. and Sacerdote, L. (1982). First passage time problems and related computational methods. Cybernetics Systems 13, 95-128.

[8] Gerstner, W. AND Kistler, W. (2002). Spiking Neuron Models. Cambridge University Press. 
[9] Giraudo, M., Sacerdote, L. and Zucca, C. (2001). A Monte Carlo method for the simulation of first passage time diffusion processes. Method. Comput. Appl. Prob. 3, 215-231.

[10] Goldman, M. (1971). On the first passage of the integrated Wiener process. Ann. Mat. Statist. 42, $2150-2155$.

[11] Groeneboom, P. (1989). Brownian motion with parabolic drift and Airy functions. Prob. Theory Relat. Fields 81, 79-109.

[12] Karatzas, I. And Shreve, S. (1987). Brownian Motion and Stochastic Calculus. Springer, New York.

[13] Kloeden, P. and Platen, E. (1992). Numerical Solution of Stochastic Differential Equations. Springer, Berlin.

[14] Lachal, A. (1991). Sur le premier instant de passage de l'intégrale du mouvement brownien. Ann. Inst. H. Poincaré Prob. Statist. 27, 385-405.

[15] Lachal, A. (1996). Quelques martingales associées à l'intégrale du processus d'Ornstein-Uhlenbeck. Application à l'étude des premiers instants d'atteinte. Stoch. Stoch. Rep. 58, 285-302.

[16] Lachal, A. (1997). Les temps de passages successifs de l'intégrale du mouvement brownien. Ann. Inst. H. Poincaré Prob. Statist. 33, 1-36.

[17] Lefebvre, M. (1989). First-passage densities of a two-dimensional process. SIAM J. Appl. Math. 49, 1514-1523.

[18] McKean, H. P. (1963). A winding problem for a resonator driven by a white noise. J. Math. Kyoto Univ. 2, 227-235.

[19] Nardo, E. D., Nobile, A. G., Pirozzi, E. and Ricciardi, L. M. (2001). A computational approach to first passage time problems for Gauss-Markov processes. Adv. Appl. Prob. 33, 453-482.

[20] Niederreiter, H. (1992). Random Number Generation and Quasi-Monte Carlo Methods. Society for Industrial and Applied Mathematics, Philadelphia, PA.

[21] Nobile, A. G., Ricciardi, L. M. and Sacerdote, L. (1985). Exponential trends of first-passage-time densities for a class of diffusion processes with steady-state distribution. J. Appl. Prob. 22, 611-618.

[22] Nobile, A. G., Ricciardi, L. M. ANd SACERdote, L. (1985). Exponential trends of Ornstein-Uhlenbeck firstpassage-time densities. J. Appl. Prob. 22, 360-369.

[23] Novikov, A., Frishling, V. And Kordzakhia, N. (1999). Approximations of boundary crossing probabilities for a Brownian motion. J. Appl. Prob. 36, 1019-1030.

[24] Patie, P. (2004). On some first passage time problem motivated by financial applications. Doctoral thesis, ETH Zurich.

[25] Pötzelberger, K. And Wang, L. (2001). Boundary crossing probability for Brownian motion. J. Appl. Prob. 38, 152-164.

[26] Ricciardi, L. M. and SATo, S. (1988). First-passage time density and moments of the Ornstein-Uhlenbeck process. J. Appl. Prob. 25, 43-57.

[27] Ricciardi, L., Sacerdote, L. And Sato, S. (1984). On an integral equation for first passage time probability density function. J. Appl. Prob. 21, 302-314.

[28] Ripley, B. D. (1987). Stochastic Simulation. John Wiley, New York.

[29] Stoer, J. And Burlisch, R. (1980). Introduction to Numerical Analysis. Springer, New York.

[30] Touboul, J. and Faugeras, O. (2007). The spikes trains probability distributions: a stochastic calculus approach. J. Physiol. Paris 101, 78-98.

[31] Wang, L. And Pötzelberger, K. (1997). Boundary crossing probability for Brownian motion and general boundaries. J. Appl. Prob. 34, 54-65.

[32] Wang, L. And Pötzelberger, K. (2007). Crossing probabilities for diffusion processes with piecewise continuous boundaries. Methodol. Comput. Appl. Prob. 9, 21-40. 